\title{
Currents induced by long waves propagating towards a beach over a wavy bed
}

\author{
By H. KYOTOH ${ }^{1}$, S. FUJII ${ }^{2}$ AND D. V. TO $\mathrm{TO}^{3}$ \\ ${ }^{1}$ Institute of Engineering Mechanics and Systems, University of Tsukuba, Tsukuba, \\ Ibaraki, 305-8573, Japan \\ ${ }^{2}$ Toyo Kokusai Oil Co., Ltd, Nagoya, Aichi, 467-0832, Japan \\ ${ }^{3}$ Department of Geophysics, Ho Chi Minh City University of Natural Science, \\ Ho Chi Minh City, Vietnam
}

(Received 23 March 1999 and in revised form 17 January 2000)

For the understanding of longshore currents along a natural beach, the effects of bottom unevenness are considered to be important, especially for the flow in the swash zone. Currents in the swash zone are strongly influenced by the bed slope because the effect of gravity overwhelms the effect of the depth change. In the present paper, we investigate these effects and focus on waves propagating from offshore over a flat ocean basin of constant depth to a beach with a sloping wavy bottom. The waves are incident at a small angle to the beach normal, and the bed slope in the alongshore direction is varied slowly. To simplify the problem, only cnoidal waves and solitary waves are considered and the bed level is varied sinusoidally in the longshore direction.

A perturbation method is applied to the two-dimensional nonlinear shallow water equation (two-dimensional NLSWE) for the wave motion in order to generate a more simplified model of wave dynamics consisting of a one-dimensional NLSWE for the direction normal to the beach and an equation for the alongshore direction. The first equation, the one-dimensional NLSWE, is solved by Carrier \& Greenspan's transformation. The solution of the second one is found by extending Brocchini \& Peregrine's solution for a flat beach. Two methods for the solution of the onedimensional NLSWE are introduced in order to get a solution applicable to largeamplitude swash motions, where the amplitude is comparable to the beach length. One is the Maclaurin expansion of the solution around the moving shoreline, and the other is Riemann's representation of the solution, which exactly satisfies the onedimensional NLSWE and the boundary conditions. After doing a consistency check by confirming that Riemann's method, a numerical solution, agrees with the exact solution for an infinitely long, sloping beach, we assumed that the Maclaurin series solution can also describe wave motion in the swash zone properly not only for this model but also for our 'wavy', finite beach model.

The solution obtained from the Maclaurin series is then plugged into the equation for the alongshore direction to calculate the shore currents induced by wave run-up and back-wash motions, where a 'weakly two-dimensional solution' is derived from geometrical considerations. The results show that since the water depth near the shoreline is comparable to the bed level fluctuations, the flow is strongly affected by the bed unevenness, leading to recognizable changes in shoreline movement and the time-averaged velocity and the mass flux of the flow in the swash zone. More specifically, the inhomogeneity of the alongshore mass flux generates offshore currents because of the continuity condition for the fluid mass. 


\section{Introduction}

An understanding of the flow in the swash zone, i.e. the area of the beach where the waves displace the instantaneous shoreline back and forth, is of importance in understanding and controlling coastal erosion and accretion. Shore currents in the swash zone have been under intensive investigation recently in order to reveal the sediment transport processes of a beach. Mass and momentum transfer in this region for a smooth uniformly sloping beach has similar properties to the Stokes' drift for progressing waves in the open ocean, as discussed in Brocchini \& Peregrine (1996) and Brocchini (1997). However, the flow over a natural beach with a spatially fluctuating elevation, i.e. a 'wavy' beach, displays a different flow pattern with cells of circular drifting flow because the gravity effects due to the fluctuations of elevation dominate the flow fields, especially near the shoreline.

Run-up and back-wash of normal incident waves have been studied by many researchers both numerically and analytically. In the following three paragraphs, we briefly review some previous research done on three different beach models with no 'wavy' beaches. The first two models are one-dimensional with the beach first treated as a virtually infinite line with a uniform slope and then as a uniformly sloping line segment with an ocean basin of constant depth. The third model is two-dimensional with the beach depicted with a uniform slope terminating in a flat, horizontal ocean basin.

For the one-dimensional 'infinite-line model', Stoker (1948) found a change of variables by which the one-dimensional nonlinear shallow water equation (onedimensional NLSWE) is transformed into a linear equation. Carrier \& Greenspan (1958) chose to use an auxiliary dependent variable in Stoker's transformation so that they could generate analytical solutions for some initial and boundary conditions. Analytical studies of a single bore and its run-up on a beach were done by Ho \& Meyer (1962) and Shen \& Meyer (1962).

For the one-dimensional flat ocean basin model with a uniformly sloping beach, Kim, Liu \& Liggett (1983) calculated solitary wave run-up and propagation applying the boundary element method. Zelt (1991) numerically solved the Boussinesq equation described in a Lagrangian coordinate system, where the friction and the dissipation terms were considered. Synolakis (1987) utilized Carrier \& Greenspan's (1958) transformation in the calculation of a solitary wave run-up, where nonlinearity was eliminated in the offshore region and the amplitude of the fluctuations of the shoreline was considered to be small compared to the slope length. Subsequently, Synolakis, Deb \& Skjelbreia (1988) derived an analytical formula illustrating the cnoidal wave run-up.

For the two-dimensional flat ocean basin model with a uniformly sloping beach, Ryrie (1983) derived a simplified model, which was decomposed into one equation for the offshore direction and one for the alongshore direction, for the study of quasi-normally incident waves over a flat bed and of the induced current in the swash zone. The equation suggested by Ryrie has been adopted by many researchers to study the generation of currents due to wave motion. For example, Asano (1994) and Kobayashi \& Karjadi (1994) obtained numerical solutions of Ryrie's equation and compared it with experimental data. Brocchini \& Peregrine (1996) extended Carrier \& Greenspan's solution (1958) for the case of obliquely incident waves. Brocchini (1997) discussed longshore mass flux and drift on a uniform sloping beach.

However, for the understanding of longshore currents along a natural beach, i.e. a 'wavy' beach, the effects of bottom unevenness are considered to be important especially for the flow in the swash zone. In the present paper, we investigate 
these effects and focus on waves propagating from offshore over an ocean basin of constant depth to a beach with a wavy bottom. In addition, discussions are restricted to obliquely incident long waves of small incident angle to the beach normal, where the two-dimensional NLSWE and the nonlinear Boussinesq equation are used as the governing equations at the beach and offshore, respectively.

In $\S 2.1$, the two-dimensional NLSWE for a 'wavy beach' is reduced by a perturbation method to more simplified equations consisting of a one-dimensional NLSWE for the direction normal to the beach and an equation for the alongshore direction. The multiple-scale perturbation method is applied to the two-dimensional NLSWE, which includes gravitational terms due to the bed slope of the beach normal and bed fluctuations in the alongshore direction. The solution of the first equation is represented in terms of Carrier \& Greenspan's transformation. The second equation is an extension of Ryrie's equation to a wavy bottom and its solution is found by extending Brocchini \& Peregrine's solution for a flat beach.

In $\S 2$ and $\S 3$, we tackle the one-dimensional NLSWE. The matching condition between the solution of the one-dimensional NLSWE and the cnoidal wave solution is illustrated, and the governing parameters of its run-up motions are introduced in $\S 2.2$. In $\S 3.1$, the one-dimensional NLSWE is solved analytically to give the Maclaurin series solution for a uniformly sloping beach. In $\S 3.2$, Riemann's representation for hyperbolic equations is applied to derive an integral equation governing wave motion at the edge of the slope. Comparisons are made between the Maclaurin series solution of $\S 3.1$ and the numerical solution of the integral equation.

In $\S 4.1$, we introduce the similarity transformation of the spatial and temporal variables by which the one-dimensional NLSWE is extended to be applicable to the two-dimensional flow. Also, the equation for the alongshore direction, which is composed of Ryrie's equation and a gravitational term due to bed fluctuations, is solved by utilizing geometrical considerations. In $\S 4.2$, the Maclaurin series solution derived in $\S 3.1$ is utilized for evaluation of currents induced by wave run-up and back-wash processes. It is emphasized in this section that the mass transport, the timeaveraged velocity and the mean bottom friction fields are very different in character from each other, particularly at the lower part of the swash zone. Throughout this paper, wave run-up and longshore currents are discussed stressing the differences in character of these fields between those for cnoidal waves for which the Eulerian mean velocity is zero and those for solitary waves.

\section{Governing equations}

In this paper, currents over a sinusoidal bed in the swash zone, as shown in figure 1 , are studied. We use the two-dimensional NLSWE for a uniformly sloping beach. Offshore, for a seabed of constant depth, the nonlinear long-wave equation with a dispersion term obtained from Boussinesq theory (see Mei 1983) is used as the governing equation. Hence, the solutions for the beach and for the offshore region are determined separately and matched with each other.

\subsection{Equations for the beach}

Ryrie (1983) developed a quasi-normal incident wave theory for a uniformly sloping beach. In the present work, a multiple-scale perturbation method is applied to the two-dimensional NLSWE after using the scaling of the variables suggested by her, and we extend Ryrie's model to include a variable bed topography. The two-dimensional 


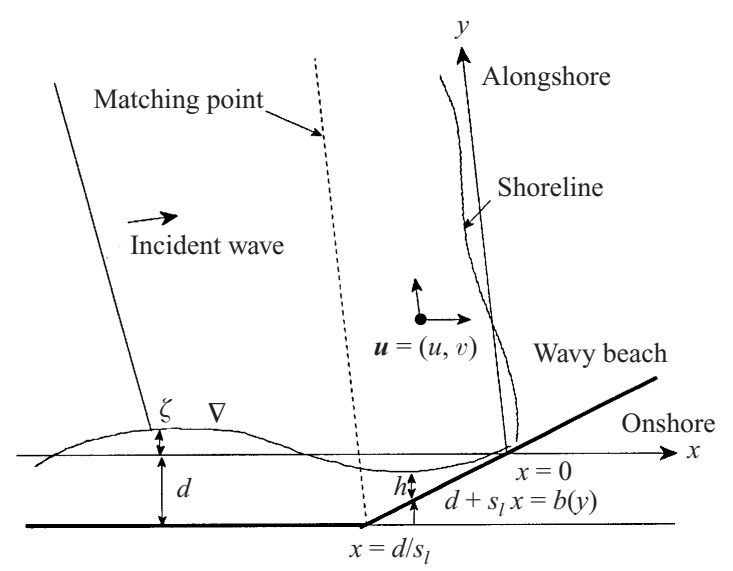

FIGURE 1. Schematic of the coordinate system and parameters.

NLSWE without friction terms are given as follows:

$$
\begin{gathered}
\frac{\partial h}{\partial t}+\frac{\partial h u}{\partial x}+\frac{\partial h v}{\partial y}=0, \\
\frac{\partial u}{\partial t}+u \frac{\partial u}{\partial x}+v \frac{\partial u}{\partial y}=-g \frac{\partial}{\partial x}\left(d+h+s_{l} x+b(y)\right), \\
\frac{\partial v}{\partial t}+u \frac{\partial v}{\partial x}+v \frac{\partial v}{\partial y}=-g \frac{\partial}{\partial y}\left(d+h+s_{l} x+b(y)\right),
\end{gathered}
$$

where $t$ is the time, $x$ and $y$ are the horizontal coordinates of the onshore and the alongshore directions, respectively, and $u$ and $v$ denote the depth-averaged flow velocity in the $x$ - and $y$-directions, respectively; $g$ is the gravitational acceleration. In addition, $d$ and $h$ are the depth of the water in the horizontal ocean basin and at the sloping beach, respectively. The function $b(y)$ is the fluctuation of the bed level from $z=s_{l} x$, where $s_{l}$ is the slope of the beach and $z$ is taken vertically upward. Here, waves are incident from $x=-\infty$, the origin of the $x$-coordinate is taken at the shoreline of the still water, and $\zeta$ is the free-surface displacement from the still water level. Hence, $\zeta$ offshore and on the sloping beach is given by $h-d$ and $h+s_{l} x+b(y)$, respectively, as shown in figure 1 .

We consider a bed fluctuation satisfying a scaling $b(y) \ll d$, which implies $0 \leqslant$ $h / b(y) \leqslant \infty$ because the depth $h$ varies from $d$ to 0 depending on the distance from the shoreline. Though there is a discontinuity of the bed level at the bottom edge of the beach, i.e. the boundary of the beach and the ocean basin, since $b(y) \neq 0$ at $x=-d / s_{l}$, the discontinuity can be assumed to influence the wave propagation only roughly to a magnitude of $b(y) / d$ because a discontinuity of the bed level in a horizontal ocean basin affects the linear long-wave propagation to the order of the step height divided by the total depth (see for instance, Mei 1983, p. 120).

We consider waves with a small incident angle to the $x$-direction and over an uneven bed having an infinitesimal slope prescribed by

$$
O\left(\frac{v}{u}\right)=O\left(\frac{\mathrm{d} b}{\mathrm{~d} y}\right)=\epsilon,
$$

where $\epsilon$ is the perturbation parameter representing the order of magnitude of the 
incident angle of the waves. Equation (2.2) implies that the perturbation expansion of the solution to (2.1) requires that

$$
\begin{gathered}
h=h_{0}+\epsilon h_{1}+\epsilon^{2} h_{2}+\cdots, \quad b=b_{0}, \\
u=u_{0}+\epsilon u_{1}+\epsilon^{2} u_{2}+\cdots, \quad v=\epsilon v_{1}+\epsilon^{2} v_{2}+\cdots .
\end{gathered}
$$

The ratio $h_{0} / b_{0}$ varies from 0 to $\infty$ depending on the offshore distance from the shoreline. Therefore, $b$ has not been scaled by $\epsilon$. The fluctuating bed level $b$ is assumed to be a function of a slow variable $\epsilon y$, as implied by (2.2). Therefore, a multiple-scale perturbation is introduced and all the independent variables are assumed to depend on the variables

$$
t, x, y_{1}=\epsilon y, \ldots,
$$

so that the secular terms in the perturbation equations can be removed.

Then, the zeroth-order perturbation equations become

$$
\frac{\partial h_{0}}{\partial t}+u_{0} \frac{\partial h_{0}}{\partial x}+h_{0} \frac{\partial u_{0}}{\partial x}=0, \frac{\partial u_{0}}{\partial t}+u_{0} \frac{\partial u_{0}}{\partial x}+g \frac{\partial h_{0}}{\partial x}+g s_{l}=0,
$$

which coincide with the one-dimensional NLSWE. But, the variables $u_{0}$ and $h_{0}$ are weakly dependent on the variable $y$ through the boundary condition at the edge of the sloping beach since the waves are incident quasi-normally to the beach.

The next-order equations are given as follows:

$$
\begin{gathered}
\frac{\partial h_{1}}{\partial t}+u_{0} \frac{\partial h_{1}}{\partial x}+\frac{\partial h_{0}}{\partial x} u_{1}+h_{0} \frac{\partial u_{1}}{\partial x}+\frac{\partial u_{0}}{\partial x} h_{1}=0, \\
\frac{\partial u_{1}}{\partial t}+u_{0} \frac{\partial u_{1}}{\partial x}+\frac{\partial u_{0}}{\partial x} u_{1}+g \frac{\partial h_{1}}{\partial x}=0, \\
\frac{\partial v_{1}}{\partial t}+u_{0} \frac{\partial v_{1}}{\partial x}=-g\left(\frac{\partial h_{0}}{\partial y_{1}}+\frac{\partial b_{0}}{\partial y_{1}}\right) .
\end{gathered}
$$

The right-hand side of $(2.6 c)$ is the external force, which can be determined by (2.5). Following the multiple-scale perturbation technique, the functional forms of $h_{0}$ and $u_{0}$ in (2.5) with respect to the variable $y_{1}$ have to be determined for $v_{1}$ in (2.6) to obtain a physically acceptable solution. This solution is derived in $\S 4.1$ as a weakly two-dimensional solution. Because the equations for $h_{1}$ and $u_{1}$ given by (2.6) have no external forces, we set $h_{1}$ and $u_{1}$ to zero. Then, the next-order equations reduce to

$$
\begin{gathered}
\frac{\partial h_{2}}{\partial t}+\frac{\partial}{\partial x}\left(u_{0} h_{2}+u_{2} h_{0}\right)+\frac{\partial}{\partial y_{1}}\left(h_{0} v_{1}\right)=0, \\
\frac{\partial u_{2}}{\partial t}+\frac{\partial}{\partial x}\left(u_{0} u_{2}+g h_{2}\right)+v_{1} \frac{\partial u_{0}}{\partial y_{1}}=0,
\end{gathered}
$$

which are used in $\S 4.2$ for the evaluation of the mass flux.

In the following, some remarks are given regarding the shoreline boundary conditions. The boundary conditions at the shore $x=x_{S}(t, y)$ are given by

$$
h=0, \quad \frac{\partial x_{S}}{\partial t}-u+v \frac{\partial x_{S}}{\partial y}=0 \quad \text { at } \quad x=x_{S}(t, y) .
$$

The first condition means that the water depth at the shoreline is identical to 0 , and the second one governs the shoreline changes induced by the flow velocity. It is shown 
from the identity

$$
\begin{aligned}
\frac{\mathrm{d}}{\mathrm{d} t} h\left(t, x_{S}, y\right)+v\left(t, x_{S}, y\right) & \frac{\mathrm{d}}{\mathrm{d} y} h\left(t, x_{S}, y\right)+h\left(t, x_{S}, y\right)\left(\frac{\partial u}{\partial x}+\frac{\partial v}{\partial y}\right) \\
= & \frac{\partial h}{\partial x}\left(\frac{\partial x_{S}}{\partial t}-u+v \frac{\partial x_{S}}{\partial y}\right)+\left(\frac{\partial h}{\partial t}+\frac{\partial h u}{\partial x}+\frac{\partial h v}{\partial y}\right),
\end{aligned}
$$

that $(2.8 b)$ is deduced from $(2.8 a)$ and the mass conservation law indicated in the first of (2.1). Therefore, these three equations are not independent. Note that the operators $\mathrm{d} / \mathrm{d} t$ and $\mathrm{d} / \mathrm{d} y$ in (2.9) are total derivatives and that $x_{S}$ is considered to be a function of $t$ and $y$.

As a result, $x_{S}$ is determined from the condition $h\left(t, x_{S}, y\right)=0$ alone, provided that a regular solution $(h, u)$ of $(2.1)$ is obtained. The perturbation system is completely determined by this condition as seen in $\S 3$. Substitution of the expansion

$$
x_{S}(t, y)=x_{S 0}\left(t, y_{1}\right)+\epsilon^{2} x_{S 2}\left(t, y_{1}\right)+O\left(\epsilon^{3}\right)
$$

and (2.3) into the kinematic boundary condition (2.8) yields the perturbation equations of the zeroth- and the second-order as follows:

$$
\begin{gathered}
h_{0}=0, \quad \frac{\partial x_{S 0}}{\partial t}-u_{0}=0 \quad \text { at } x=x_{S 0}, \\
h_{2}+\frac{\partial h_{0}}{\partial x} x_{S 2}=0, \quad \frac{\partial x_{S_{2}}}{\partial t}-u_{2}+\frac{\partial u_{0}}{\partial x} x_{S 2}+v_{1} \frac{\partial x_{S 0}}{\partial y_{1}}=0 \quad \text { at } \quad x=x_{S 0} .
\end{gathered}
$$

Here the first-order term of $x_{S}$ with respect to $\epsilon$ has been eliminated in (2.10) because $h_{1}=0$ and $u_{1}=0$. Consequently, only the first condition of each equation in (2.11) is needed to determine all the unknown variables.

\subsection{Equations for the offshore region}

Although the dispersion of long waves can be ignored on the sloping beach, it is important for the offshore region. Hence, the nonlinear Boussinesq equation is used for our governing equation in this region. According to Mei (1983), Boussinesq theory leads to the following weakly nonlinear shallow water equation:

$$
\begin{gathered}
\frac{\partial \zeta}{\partial t}+\nabla \cdot(h \boldsymbol{u})=0, \\
\frac{\partial \boldsymbol{u}}{\partial t}+\boldsymbol{u} \cdot \nabla \boldsymbol{u}+g \nabla \zeta-\frac{d^{2}}{3} \nabla \nabla \cdot \frac{\partial \boldsymbol{u}}{\partial t}=0 .
\end{gathered}
$$

Here $\boldsymbol{u}$ is a velocity vector defined by $(u, v), \zeta$ is a free-surface displacement described in figure 1 , and $\nabla$ denotes the gradient operator of the spatial variables $x$ and $y$. Since we are considering quasi-normally-incident, weakly-nonlinear long waves propagating to the beach, the waves offshore can be described by a linear superposition of the incident and the reflected waves, as shown in Miles (1977). Therefore, in this section, the solution of the one-dimensional waves is derived first and then is extended to the case of oblique incidence later in $\S 4$. Since derivations of the following equations can be found in the book written by Mei (1983), only results are shown here. Assuming that the ratio between wave amplitude and depth offshore is small, the solution of (2.12) can be expanded as a series

$$
\boldsymbol{u}=\varepsilon \boldsymbol{u}^{(1)}+\varepsilon^{2} \boldsymbol{u}^{(2)}+\cdots, \quad \zeta=\varepsilon \zeta^{(1)}+\varepsilon^{2} \zeta^{(2)}+\cdots,
$$


where $\varepsilon$ is the perturbation parameter for the wave amplitude. Under the Boussinesq scaling, the solution is given as follows:

$$
\zeta^{(1)}=\zeta_{I}^{(1)}+\zeta_{R}^{(1)}, \quad u^{(1)}=\frac{c_{0}}{d}\left(\zeta_{I}^{(1)}-\zeta_{R}^{(1)}\right), \quad c_{0} \equiv \sqrt{g d},
$$

where $\zeta_{I}^{(1)}$ and $\zeta_{R}^{(1)}$ denote the incident and the reflected waves, respectively. These are governed by the $\mathrm{KdV}$ equation

$$
\begin{aligned}
& \frac{\partial \zeta_{I}^{(1)}}{\partial t}+c_{0} \frac{\partial \zeta_{I}^{(1)}}{\partial x}+\frac{3 g}{2 \sqrt{g d}} \zeta_{I}^{(1)} \frac{\partial \zeta_{I}^{(1)}}{\partial x}+\frac{d^{2} \sqrt{g d}}{6} \frac{\partial^{3} \zeta_{I}^{(1)}}{\partial x^{3}}=0, \\
& \frac{\partial \zeta_{R}^{(1)}}{\partial t}-c_{0} \frac{\partial \zeta_{R}^{(1)}}{\partial x}-\frac{3 g}{2 \sqrt{g d}} \zeta_{R}^{(1)} \frac{\partial \zeta_{R}^{(1)}}{\partial x}-\frac{d^{2} \sqrt{g d}}{6} \frac{\partial^{3} \zeta_{R}^{(1)}}{\partial x^{3}}=0 .
\end{aligned}
$$

The reflected wave $\zeta_{R}^{(1)}$ can be determined by a matching of the free-surface displacement at the boundary between the offshore region and the sloping beach. It is useful for the calculation of the wave motion on the sloping beach to derive a boundary condition at the end of the slope, which does not involve the reflected wave component. Elimination of the reflected wave component in (2.14) between the two equations in (2.14) yields the following relation:

$$
\zeta^{(1)}+\frac{d}{c_{0}} u^{(1)}=2 \zeta_{I}^{(1)} \quad \text { at } \quad x=-r_{b}
$$

where $r_{b}=d / s_{l}$ denotes the distance between the shoreline of the still water and the end of the sloping beach. The boundary condition for the waves in the sloping beach is given by considering the continuity of the free-surface level and the flow velocity at this boundary.

In the present study, Wiegel's formula for the cnoidal wave solution (Wiegel 1960)

$$
\begin{gathered}
\zeta_{I}^{(1)}=\frac{H}{\mu}\left(1-\mu-\frac{E}{K}\right)+H \mathrm{cn}^{2}\left[\frac{2 K}{L}(x-C t)\right], \\
C^{2}=g d\left\{1+\frac{H}{\mu d}\left(-\mu+2-3 \frac{E}{K}\right)\right\}, \\
L=4 K d \sqrt{\frac{\mu d}{3 H}},
\end{gathered}
$$

is adopted to give the incident wave. Here, $H$ is the wave height, $C$ is the wave celerity, $L$ is the wavelength, $\mathrm{cn}$ is the cosine-elliptic function, and $K$ and $E$ denote the complete elliptic integrals of the first kind and the second kind, which are defined by

$$
K=\int_{0}^{\pi / 2}\left(1-\mu \sin ^{2} \chi\right)^{-1 / 2} \mathrm{~d} \chi, \quad E=\int_{0}^{\pi / 2}\left(1-\mu \sin ^{2} \chi\right)^{1 / 2} \mathrm{~d} \chi
$$

respectively. Finally, $\mu$ is a parameter related to the wave profile (see also Mei 1983).

There are three non-dimensional parameters which govern the run-up phenomena. Applying non-dimensionalization to (2.1) using $r_{b}, \sqrt{r_{b} /\left(g s_{l}\right)}$ and $\sqrt{g s_{l} r_{b}}$ as the characteristic length, time, and velocity scales, the transformed equation (2.1) for the unknowns $h /\left(s_{l} r_{b}\right), u / \sqrt{g s_{l} r_{b}}$, and $v / \sqrt{g s_{l} r_{b}}$ does not explicitly include any governing parameters (see, for example, Brocchini \& Peregrine 1996). Therefore, the governing parameters of the wave motion appear only in the boundary condition (2.16), which 
is described by

$$
\begin{aligned}
& \left.\left(\frac{\zeta^{(1)}}{s_{l} r_{b}}+\frac{u^{(1)}}{\sqrt{g s_{l} r_{b}}}\right)\right|_{x / r_{b}=-1} \\
& \quad=2 \frac{H}{d}\left\{\frac{1}{\mu}\left(1-\mu-\frac{E}{K}\right)+\operatorname{cn}^{2}\left[2 K\left(-\frac{r_{b}}{L}-\frac{C}{L} \sqrt{\frac{r_{b}}{g s_{l}}} \frac{t}{\sqrt{r_{b} /\left(g s_{l}\right)}}\right)\right]\right\} .
\end{aligned}
$$

Since the phase of the cnoidal function at $t=0$ is only related to the translation of the time, it is ignored for the stationary motion. This equation reveals that the three non-dimensional parameters

$$
\mu, \quad \frac{H}{d}, \quad K \frac{C}{L} \sqrt{\frac{r_{b}}{g s_{l}}} \equiv \kappa
$$

govern the run-up and back-wash motion. The first two parameters are related to the incident cnoidal wave and the last one represents the wave characteristics at the slope.

\section{Analytical expressions for the solution for the beach}

Before studying the two-dimensional wave motions, the one-dimensional problem governed by (2.5) needs to be solved under the boundary condition (2.16). Extension of the one-dimensional problem to the two-dimensional one is achieved by the geometrical considerations introduced in the next section.

Here, two methods to derive solutions of non-breaking waves in (2.5) are introduced. The first is a series solution, which is applied for large-amplitude swash motions. The second is an exact integral equation derived by Riemann's representation of hyperbolic equations (Courant \& Hilbert 1965). The numerical solution of Riemann's representation is compared with the series solution to assess the truncation error of the series.

\subsection{Series solution}

In our model, the beach has a slope smaller than $1 / 10$ and a length shorter than the wavelength of the non-breaking long waves incident to the beach. Therefore, it is appropriate to use the series expansion of Carrier \& Greenspan's transformation, which is applicable to the case of large-amplitude swash motions.

Carrier \& Greenspan's (1958) solution of (2.5) is given by

$$
u_{0}=\frac{m}{\sigma} \frac{\partial \phi}{\partial \sigma}, \quad h_{0}=\frac{s_{l}}{16 m} \sigma^{2}, \quad x=-\frac{\sigma^{2}}{16 m}+\frac{1}{4} \frac{\partial \phi}{\partial \lambda}-\frac{u_{0}^{2}}{2 m}, \quad t=\frac{\lambda}{2 m}-\frac{u_{0}}{m},
$$

where $m=s_{l} g$ and $\phi$ satisfies

$$
\frac{\partial}{\partial \sigma}\left(\sigma \frac{\partial \phi}{\partial \sigma}\right)-\sigma \frac{\partial^{2} \phi}{\partial \lambda^{2}}=0
$$

Here, $\lambda$ and $\sigma$ are independent variables. Taking into account that $\sigma=0$ corresponds to the shoreline and that both the velocity $u_{0}$ and the depth $h_{0}$ are represented by analytic functions at the shoreline, $\phi$ can be expanded in a Maclaurin series with 


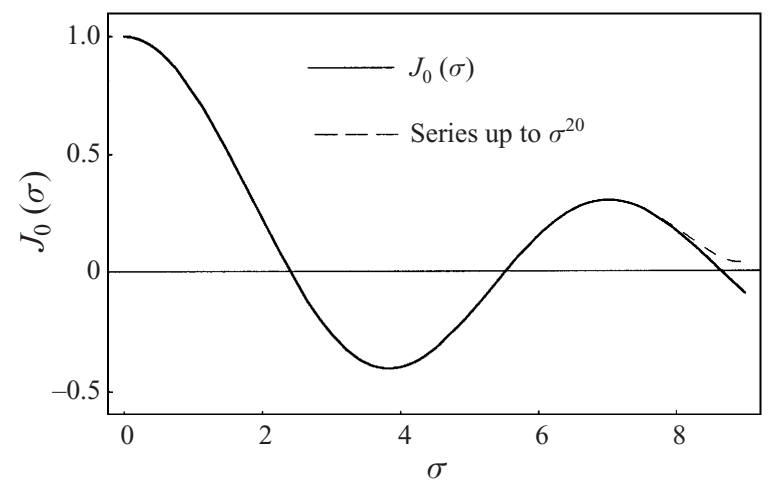

Figure 2. The Bessel function of zeroth-order $J_{0}(\sigma)$ and its truncated series.

respect to $\sigma$. Substitution of the Maclaurin series into (3.2) gives an iterative relation for the coefficients given by

$$
\phi^{(\ell)}=\frac{\ell-1}{\ell} \frac{\mathrm{d}^{2}}{\mathrm{~d} \lambda^{2}} \phi^{(\ell-2)},\left.\quad \phi^{(\ell)} \equiv \frac{\partial^{\ell}}{\partial \sigma^{\ell}} \phi\right|_{\sigma=0}
$$

where $\ell=2,4,6, \ldots, \infty$. Equation (3.3) can be manipulated to give

$$
\phi=\sum_{n=0}^{\infty} \frac{\sigma^{2 n}}{n ! 2^{2 n} \Gamma(n+1)} \frac{\mathrm{d}^{2 n} A(\lambda)}{\mathrm{d} \lambda^{2 n}},\left.\quad A(\lambda) \equiv \phi\right|_{\sigma=0},
$$

where $\Gamma$ is the Gamma function and $A(\lambda)$ is a function which is determined from the matching condition at the end of the slope. In order to examine the convergence of the series (3.4), the zeroth-order Bessel function $J_{0}(\sigma)$, which is the amplitude of an exact solution of (3.2) given by $\phi=J_{0}(\sigma) \sin \lambda$, and its truncated series with respect to $\sigma$ are depicted in figure 2. The figure suggests that the truncated series (3.4) up to order $\sigma^{20}$ is sufficient to approximate oscillations of the long waves on the uniformly sloping beach. In the present study, we will consider cnoidal waves with wavelength roughly four times the length of the sloping beach. Therefore, the following truncated series of $\phi$ :

$$
\phi \approx \phi_{a p}=\sum_{n=0}^{10} \frac{\sigma^{2 n}}{n ! 2^{2 n} \Gamma(n+1)} \frac{\mathrm{d}^{2 n} A(\lambda)}{\mathrm{d} \lambda^{2 n}},
$$

is used as an approximate solution of (3.2).

Assuming the terms proportional to the square of $A(\lambda)$ in (3.1) are small at the matching point $x=-r_{b}$ and substituting (3.5) into (3.1), the approximate values of $\zeta_{0}$, which is defined by $s_{l} x+h_{0}$, and $u_{0}$ at $x=-r_{b}$ are obtained explicitly by a perturbation method as follows:

$$
\begin{aligned}
u_{0 b} \approx & \frac{m}{2} \frac{\mathrm{d}^{2} A}{\mathrm{~d} \lambda^{2}}+m^{2} r_{b} \frac{\mathrm{d}^{4} A}{\mathrm{~d} \lambda^{4}}+\frac{2 m^{3} r_{b}^{2}}{3} \frac{\mathrm{d}^{6} A}{\mathrm{~d} \lambda^{6}}+\frac{2 m^{4} r_{b}^{3}}{9} \frac{\mathrm{d}^{8} A}{\mathrm{~d} \lambda^{8}}+\frac{2 m^{5} r_{b}^{4}}{45} \frac{\mathrm{d}^{10} A}{\mathrm{~d} \lambda^{10}}+\frac{4 m^{6} r_{b}^{5}}{675} \frac{\mathrm{d}^{12} A}{\mathrm{~d} \lambda^{12}} \\
& +\frac{8 m^{7} r_{b}^{6}}{14175} \frac{\mathrm{d}^{14} A}{\mathrm{~d} \lambda^{14}}+\frac{4 m^{8} r_{b}^{7}}{99225} \frac{\mathrm{d}^{16} A}{\mathrm{~d} \lambda^{16}}+\frac{2 m^{9} r_{b}^{8}}{893025} \frac{\mathrm{d}^{18} A}{\mathrm{~d} \lambda^{18}}+\frac{4 m^{10} r_{b}^{9}}{40186125} \frac{\mathrm{d}^{20} A}{\mathrm{~d} \lambda^{20}}
\end{aligned}
$$




$$
\begin{aligned}
\zeta_{0 b} \approx & S_{l}\left(\frac{1}{4} \frac{\mathrm{d} A}{\mathrm{~d} \lambda}+m r_{b} \frac{\mathrm{d}^{3} A}{\mathrm{~d} \lambda^{3}}+m^{2} r_{b}^{2} \frac{\mathrm{d}^{5} A}{\mathrm{~d} \lambda^{5}}+\frac{4 m^{3} r_{b}^{3}}{9} \frac{\mathrm{d}^{7} A}{\mathrm{~d} \lambda^{7}}+\frac{m^{4} r_{b}^{4}}{9} \frac{\mathrm{d}^{9} A}{\mathrm{~d} \lambda^{9}}+\frac{4 m^{5} r_{b}^{5}}{225} \frac{\mathrm{d}^{11} A}{\mathrm{~d} \lambda^{11}}\right. \\
& \left.+\frac{4 m^{6} r_{b}^{6}}{2025} \frac{\mathrm{d}^{13} A}{\mathrm{~d} \lambda^{13}}+\frac{16 m^{7} r_{b}^{7}}{99225} \frac{\mathrm{d}^{15} A}{\mathrm{~d} \lambda^{15}}+\frac{m^{8} r_{b}^{8}}{99225} \frac{\mathrm{d}^{17} A}{\mathrm{~d} \lambda^{17}}+\frac{4 m^{9} r_{b}^{9}}{8037225} \frac{\mathrm{d}^{19} A}{\mathrm{~d} \lambda^{19}}\right)
\end{aligned}
$$

where the subscript $b$ of $u_{0}$ and $\zeta_{0}$ denotes the value at $x=-r_{b}$. Here, all of the first-order terms of $A(\lambda)$ at the edge of the slope has been considered in (3.6), i.e. $\sigma \approx 4 \sqrt{m r_{b}}+O(A(\lambda))$ at $x=-r_{b}$. It is applicable to finite-amplitude swash motions, where the amplitude is comparable to the beach length. Although the above approximation leads to the elimination of the term $u_{0} / m$ at the matching point, it does not mean that the term $u_{0} / m$ has been ignored at the beach. The convergence of the truncated series (3.6) can be checked roughly by taking the ratio of the successive terms in (3.6), which yields

$$
O\left(\frac{4 m^{10} r_{b}^{9}}{40186125} \frac{\mathrm{d}^{20} A}{\mathrm{~d} \lambda^{20}} / \frac{2 m^{9} r_{b}^{8}}{893025} \frac{\mathrm{d}^{18} A}{\mathrm{~d} \lambda^{18}}\right)=\frac{2 m r_{b}}{45}\left(\frac{\omega}{2 m}\right)^{2} \ll 1,
$$

where we have assumed $\lambda \approx 2 m t$, and $\omega$ is the wave frequency defined by $2 \pi C / L$. Hence, the truncated series (3.5) is accurate if inequality (3.7) is satisfied. Note that the ratio in (3.7) is proportional to $\kappa^{2}$, which appears in (2.20).

If (3.6) is used as an approximate solution of $u_{0 b}$ and $\zeta_{0 b}$, the matching condition (2.16) leads to the equation of $A(\lambda)$

$$
\zeta_{0 b}+\frac{d}{c_{0}} u_{0 b}=\left.2 \zeta_{I}^{(1)}\right|_{x=-r_{b}} .
$$

To calculate the wave run-up distance, $\sigma$ is put to 0 in (3.1), giving

$$
x_{S 0}=\frac{1}{4} \frac{\mathrm{d} A}{\mathrm{~d} \lambda}-\frac{1}{2 m}\left(\frac{m}{2}\right)^{2}\left(\frac{\mathrm{d}^{2} A}{\mathrm{~d} \lambda^{2}}\right)^{2}, \quad t_{S 0}=\frac{\lambda}{2 m}-\frac{1}{2} \frac{\mathrm{d}^{2} A}{\mathrm{~d} \lambda^{2}},
$$

where $x_{S 0}$ and $t_{S 0}$ denote shore position and time along the shoreline to the zeroth order with respect to $\epsilon$, respectively. The linear approximation (3.8) at the matching point, as implied by (2.19), implies the following formulas for the maximum and minimum shoreline positions:

$$
\left.\begin{array}{l}
x_{S 0 \max }=\frac{1}{4} \max _{\lambda}\left[\frac{\mathrm{d} A}{\mathrm{~d} \lambda}\right]=\frac{H}{4 s_{l}} f_{\max }(\kappa, \mu), \\
x_{S 0 \min }=\frac{1}{4} \min _{\lambda}\left[\frac{\mathrm{d} A}{\mathrm{~d} \lambda}\right]=\frac{H}{4 s_{l}} f_{\min }(\kappa, \mu),
\end{array}\right\}
$$

where the subscripts $\max$ and $\min$ indicate the maximum and the minimum of the corresponding function, and both $f_{\max }$ and $f_{\min }$ are functions of $\kappa$ and $\mu$ which will be shown later in figure 5 . The above equality suggests that the run-up height is represented by only two non-dimensional parameters. This result is consistent with Synolakis's formula for the solitary wave run-up height (Synolakis 1987). Equation (3.10) is satisfied for waves whose nonlinearity is negligible at the end of the slope.

In order to check the accuracy of the solution to (3.8), a solitary wave run-up is calculated as an example. Applying the Fourier transformation to the differential equation (3.8) and using the solitary wave solution of (2.15), the unknown function $A(\lambda)$ is provided by a Fourier integral. Figure 3 shows the run-up distance, which is 


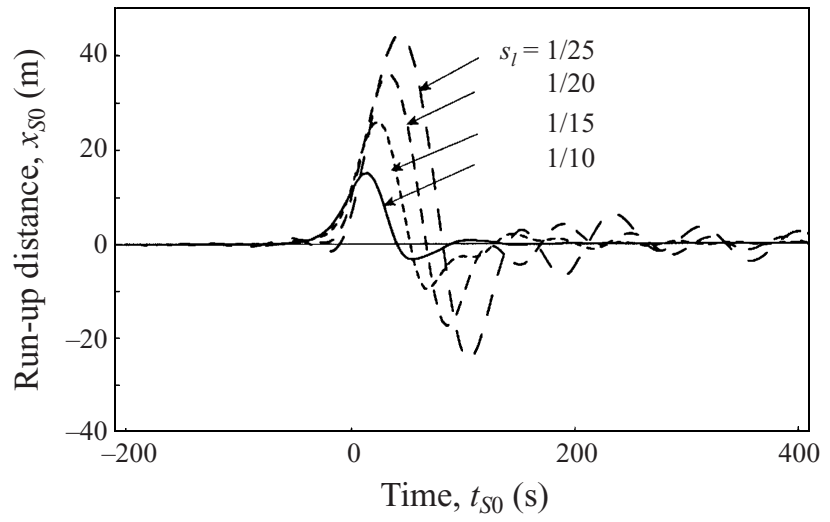

FiguRE 3. Solitary wave run-up distance $x_{S 0}$ as a function of time. Here, $d=50 \mathrm{~m}$ and $H / d=0.01$.

calculated by (3.9) for $H / d=0.01$. Here, the depth is fixed to $d=50 \mathrm{~m}, g=9.8 \mathrm{~m} \mathrm{~s}^{-2}$, and the bed slope is $s_{l}=\frac{1}{10}, \frac{1}{15}, \frac{1}{20}$, and $\frac{1}{25}$, successively. The exact expression (3.1) has been applied to evaluate the run-up distance in figure 3, as discussed by Synolakis (1987). This expression includes nonlinear terms which cause the troughs to be steeper than the crests of the oscillations in figure 3. But, both the frequency and amplitude of the tails are larger than the numerical results depicted in figure 10 shown in the next section. This discrepancy comes from the truncation error of the series (3.4) since higher-order terms of the series (3.4) are necessary to describe these short-wave components.

To examine the swash motion of periodic long waves, we modelled the incident waves on the beach as cnoidal wave with a zero Eulerian mean velocity. Putting the modulus $\mu$ at 0.99 and $H / d$ to 0.01 in (2.17), the wave period $T_{p}$ is then equal to $191.6 \mathrm{~s}$ and the wavelength $4246 \mathrm{~m}$ according to cnoidal wave theory (Wiegel 1960). Figure 4 shows the run-up height as a function of time for various bed slopes. Here, $d=50 \mathrm{~m}$ and the bed slope is $s_{l}=\frac{1}{10}, \frac{1}{15}, \frac{1}{20}$, and $\frac{1}{25}$, successively. Though the run-up height in general becomes a decreasing function of the bed slope, it seems to be more complicated than expected as indicated by Synolakis et al. (1988) because it appears as a consequence of the interaction between the incident and the resulting reflected waves. Figure 5 depicts the values of both $f_{\max }(\kappa, \mu)$ and $f_{\min }(\kappa, \mu)$ defined by (3.10) as a function of $\kappa$ for $\mu=0.9$ and 0.99 , where the run-up formula derived by Synolakis et al. (1988) is applied for the calculation. Lozenges and crosses in the figure show the data obtained from figure 4 and the numerical simulation of the next section, respectively. Although Synolakis et al. used the exact solution of (3.2), $\sigma$ at $x=-r_{b}$ was approximated by $4 \sqrt{m r_{b}}$. Hence, Synolakis's (1987) theory leads to an error in predicting swash motions, where the amplitude is not negligible compared to the beach length.

The dependence of the non-dimensional run-up height on $\mu$ for a fixed value of $\kappa$ is not strong since the parameter $\kappa$ dominates the oscillations of the flow in the beach as indicated in (2.19).

In the present study, we keep the parameters $d, H$, and $g$ as dimensional quantities in order to relate them more easily to the real situation, though non-dimensional similarity parameters exist for this phenomena as mentioned in $\S 2.2$. The solutions obtained in this section are applied to evaluations of the currents in $\S 4$. 


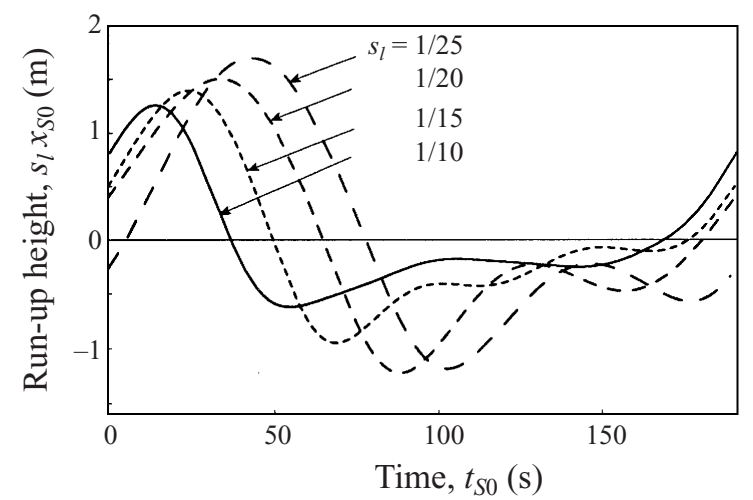

FiguRE 4. Run-up height of cnoidal wave as a function of time. Here, $d=50 \mathrm{~m}$, $H / d=0.01$, and $T_{p}=191.6 \mathrm{~s}$.

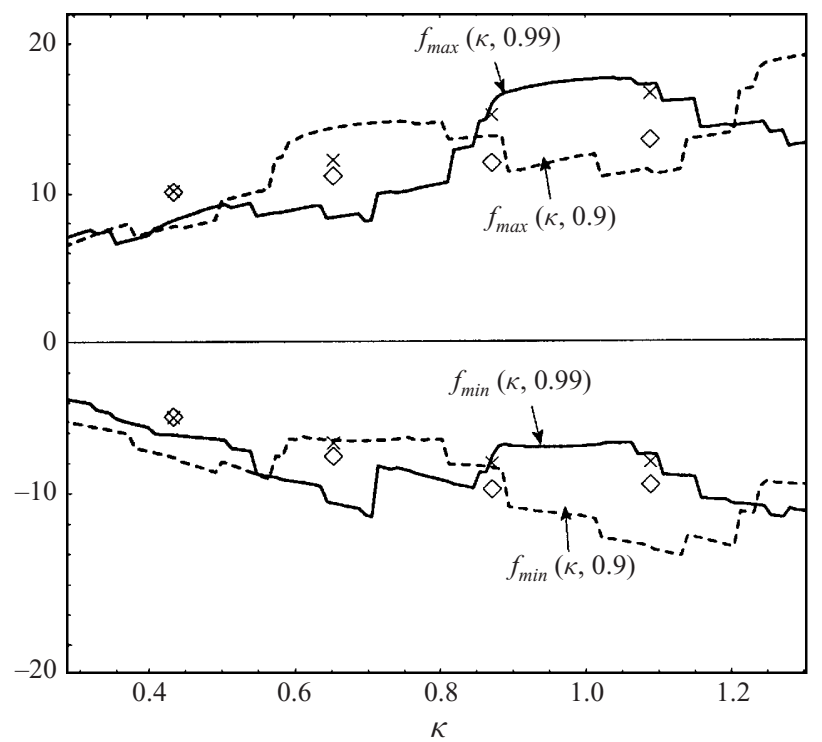

FIGURE 5. $f_{\max }$ and $f_{\min }$ as a function of $\kappa$ for $\mu=0.9$ and $\mu=0.99$, respectively. Here, lozenges and crosses denote the values obtained from figure 4 and figure 12, respectively.

In the next section, the accuracy of the present series solution is investigated with a numerical simulation.

\subsection{An exact integral formula in terms of Riemann's representation}

After substitution of (3.6) into (3.8), the resulting partial differential equation has a high enough accuracy for our purposes provided any nonlinearity of the waves at $x=-r_{b}$, i.e. the border of the beach with the ocean basin, is negligible. In this section, an exact equation, which satisfies the nonlinear matching condition at $x=-r_{b}$, is derived and calculated numerically to check the accuracy of the approximation done in the previous section. Riemann's representation of hyperbolic equations reduces the domain problem to the boundary value problem, which is composed of one integral equation and two nonlinear algebraic equations.

Consider the transformation of the variables from $\sigma$ and $\lambda$ to the characteristic 

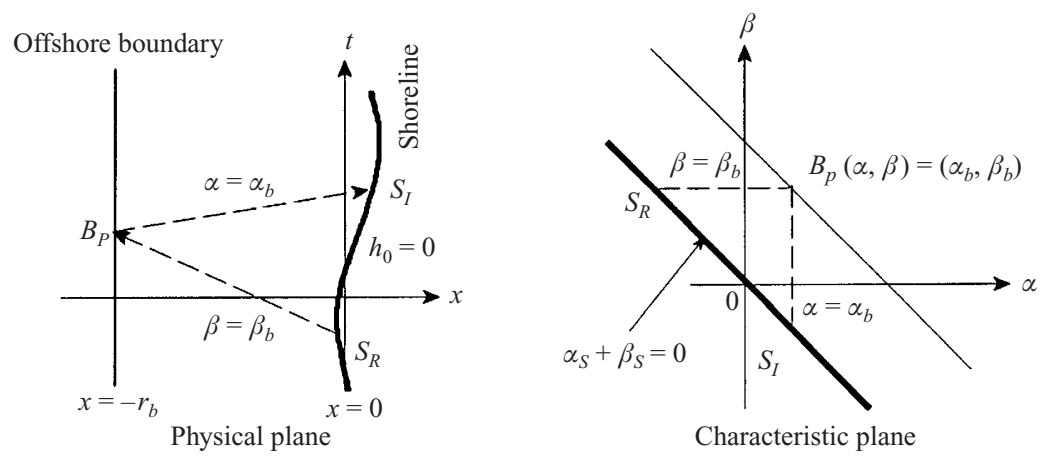

FIGURE 6. Characteristic curves of non-breaking waves.

coordinates $\alpha$ and $\beta$,

$$
\alpha=\frac{\lambda+\sigma}{2}, \quad \beta=\frac{-\lambda+\sigma}{2}, \quad \phi=\frac{Y}{\sqrt{\alpha+\beta}},
$$

where $\alpha=$ const. and $\beta=$ const. depict the incoming and the outgoing characteristics from the shoreline, respectively. Then, we have the Euler-Poisson equation for the variable $Y$ (Shen \& Meyer 1962)

$$
\frac{\partial^{2} Y}{\partial \alpha \partial \beta}+\frac{1}{4(\alpha+\beta)^{2}} Y=0
$$

Riemann's solution $R_{n}$ of (3.12) is

$$
R_{n}\left(\alpha, \beta ; \alpha_{0}, \beta_{0}\right)=\sqrt{\frac{\left(\alpha_{0}+\beta_{0}\right)(\alpha+\beta)}{\left(\alpha_{0}+\beta\right)\left(\alpha+\beta_{0}\right)}}{ }_{2} F_{1}\left[\frac{1}{2}, \frac{1}{2}, 1, \frac{\left(\alpha-\alpha_{0}\right)\left(\beta-\beta_{0}\right)}{\left(\alpha+\beta_{0}\right)\left(\beta+\alpha_{0}\right)}\right],
$$

which has been determined so as to satisfy two initial conditions given by $R_{n}\left(\alpha_{0}, \beta ; \alpha_{0}, \beta_{0}\right)=1$ and $R_{n}\left(\alpha, \beta_{0} ; \alpha_{0}, \beta_{0}\right)=1$ (Rozdestvenskii \& Janenko 1983). Here, ${ }_{2} F_{1}$ denotes the hypergeometric function.

The boundary integral method gives the relation between the values of $Y$ at the edge of the beach and the shoreline. Application of Green's theorem to (3.12) in the region $B_{P} S_{R} S_{I}$ depicted in figure 6 yields the following integral relation:

$$
\begin{aligned}
& 2 Y\left(B_{P}\right)=Y\left(S_{R}\right)+Y\left(S_{I}\right) \\
& \quad-\int_{S_{R} S_{I}}\left[\left(\frac{\partial Y}{\partial \alpha_{S}} R_{n}-Y \frac{\partial R_{n}}{\partial \alpha_{S}}\right) \frac{\mathrm{d} \beta_{S}}{\mathrm{~d} v}+\left(\frac{\partial Y}{\partial \beta_{S}} R_{n}-Y \frac{\partial R_{n}}{\partial \beta_{S}}\right) \frac{\mathrm{d} \alpha_{S}}{\mathrm{~d} v}\right] \mathrm{d} s .
\end{aligned}
$$

Here, $s$ is the distance along the curve $S_{R} S_{I}, v$ denotes its outward normal vector, and $\left(\alpha_{S}, \beta_{S}\right)$ is the point on the curve $S_{R} S_{I}$ parametrized by $s$, so that

$$
\mathrm{d} s^{2}=\mathrm{d} \alpha_{S}^{2}+\mathrm{d} \beta_{S}^{2}, \frac{\mathrm{d} \alpha_{S}}{\mathrm{~d} v}=\frac{\mathrm{d} \beta_{S}}{\mathrm{~d} s}, \frac{\mathrm{d} \beta_{S}}{\mathrm{~d} v}=-\frac{\mathrm{d} \alpha_{S}}{\mathrm{~d} s} .
$$

Moreover, due to the relation $\alpha_{S}+\beta_{S}=0$, which is identified with the boundary condition $h_{0}=0$ on the shoreline, (3.15) leads to

$$
\alpha_{S}(s)=\frac{s}{\sqrt{2}}, \quad \beta_{S}(s)=-\frac{s}{\sqrt{2}},
$$

and both $Y\left(S_{R}\right)$ and $Y\left(S_{I}\right)$ are equal to 0 provided that $\phi$ is finite at the shoreline, that 
is, for non-breaking waves. Therefore, (3.14) is regarded as the determining equation of $\phi\left(B_{P}\right)$, which reduces to

$$
\phi\left(B_{P}\right)=\frac{1}{\sqrt{2} \pi} \int_{s_{R}}^{s_{I}} \frac{\phi\left(\alpha_{S}, \beta_{S}\right)}{\sqrt{\left(\alpha_{b}+\beta_{S}\right)\left(\alpha_{S}+\beta_{b}\right)}} \mathrm{d} s,
$$

where the limit $\alpha_{S}+\beta_{S} \rightarrow 0$ is applied to (3.14). Here, the asymptotic expansion of the hypergeometric function ${ }_{2} F_{1}$ around a logarithmic singular point

$$
{ }_{2} F_{1}\left[\frac{1}{2}, \frac{1}{2}, 1, z\right]=-\frac{1}{\pi} \log |1-z|+O(1)
$$

has been used for the derivation of (3.17).

Also, $s_{R}$ and $s_{I}$ are related to the position $B_{P}\left(\alpha_{b}, \beta_{b}\right)$ by

$$
\beta_{S}\left(s_{R}\right)=\beta_{b}, \quad \alpha_{S}\left(s_{I}\right)=\alpha_{b},
$$

as shown in figure 6 . A change of the variable $s$ to $\tau$, defined by $\tau=s / \sqrt{2}$, changes (3.17) to the more general formula

$$
\phi\left(\alpha_{b}, \beta_{b}\right)=\frac{1}{\pi} \int_{-\beta_{b}}^{\alpha_{b}} \frac{\phi_{S}(\tau)}{\sqrt{\left(\beta_{b}+\tau\right)\left(\alpha_{b}-\tau\right)}} \mathrm{d} \tau, \quad \phi_{S}(\tau) \equiv \phi(\tau,-\tau),
$$

where $\phi_{S}$ denotes the value of $\phi$ at the shoreline. For numerical computations, it would be convenient to transform (3.20) into

$$
\phi\left(\alpha_{b}, \beta_{b}\right)=\frac{1}{\pi} \int_{-1}^{1} \frac{\phi_{S}(\tau(\delta ; t))}{\sqrt{1-\delta^{2}}} \mathrm{~d} \delta,
$$

where transformation of the variable $\tau$ to $\delta$ is defined as follows:

$$
\tau(\delta ; t)=\frac{\alpha_{b}+\beta_{b}}{2} \delta+\frac{\alpha_{b}-\beta_{b}}{2} .
$$

Carrier \& Greenspan's transformation at the boundary $x=-r_{b}$ gives equations for the two unknowns $\phi_{S}(\tau)$ and $\beta_{b}(t)$ as follows:

$$
\begin{aligned}
-r_{b} & =\frac{1}{8}\left(\frac{\partial \phi_{b}}{\partial \alpha_{b}}-\frac{\partial \phi_{b}}{\partial \beta_{b}}\right)-\frac{\left(\alpha_{b}+\beta_{b}\right)^{2}}{16 m}-\frac{u_{0}^{2}}{2 m}, \\
t & =\frac{\alpha_{b}-\beta_{b}}{2 m}-\frac{u_{0}}{m}
\end{aligned}
$$

where

$$
u_{0}=\frac{m}{2\left(\alpha_{b}+\beta_{b}\right)}\left(\frac{\partial \phi_{b}}{\partial \alpha_{b}}+\frac{\partial \phi_{b}}{\partial \beta_{b}}\right), \quad \phi_{b} \equiv \phi\left(\alpha_{b}, \beta_{b}\right),
$$

where the partial derivatives of $\phi_{b}$ are given by

$$
\left.\begin{array}{l}
\frac{\partial \phi_{b}}{\partial \alpha_{b}}=\frac{1}{2 \pi} \int_{-1}^{1} \sqrt{\frac{1+\delta}{1-\delta}} \phi_{S}^{\prime}(\tau(\delta ; t)) \mathrm{d} \delta, \\
\frac{\partial \phi_{b}}{\partial \beta_{b}}=\frac{-1}{2 \pi} \int_{-1}^{1} \sqrt{\frac{1-\delta}{1+\delta}} \phi_{S}^{\prime}(\tau(\delta ; t)) \mathrm{d} \delta .
\end{array}\right\}
$$

Here, we have used a partial integration so that the kernels of these integrals have a weak singularity. Note that $\alpha_{b}$ is given as a function of $t$ for the subcritical flow because it is conserved on the incoming characteristics (Carrier \& Greenspan 1958). 


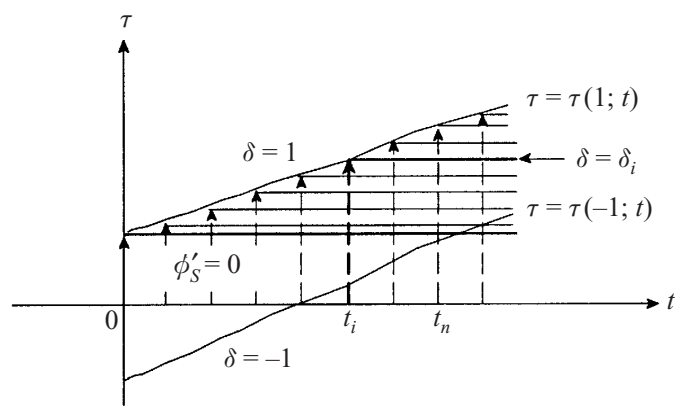

FIGURE 7. Graph of $\tau=\tau(\delta ; t)$ and mesh of integration in (3.24).

In this case, $\beta_{b}(t)$ and $\phi_{S}^{\prime}(\tau(\delta ; t))$ are the unknowns and can be determined by their past data through (3.23) and (3.24).

Consider an incident wave propagating from offshore to the still water on the beach. There is a difficulty in solving (3.24) numerically because the variable $\tau$ of $\phi_{S}^{\prime}$ depends on both $\delta$ and $t$. It is resolved, however, if the following variable mesh is introduced for the numerical integration. Assuming that $\phi_{S}^{\prime}\left(\tau\left(1 ; t_{i}\right)\right)$, where $i=0, \ldots, n-1$, are given, then the numerical integration in (3.24) is performed under mesh points $\delta=\delta_{i}, i=0, \ldots, n-1$, which are specified by

$$
\tau\left(\delta_{i} ; t_{n}\right)=\tau\left(1 ; t_{i}\right)
$$

as shown in figure 7 .

In order to calculate $\phi_{S}^{\prime}\left(\tau\left(1 ; t_{n}\right)\right)$ and $\beta_{b}\left(t_{n}\right)$, the successive over-relaxation (SOR) method (see for instance, Lapidus \& Pinder 1982) is applied since (3.23) is highly nonlinear with respect to $\beta_{b}$. At the first iteration, $u_{0}$ of (3.23) is given an initial value, then $\beta_{b}$ is calculated by (3.23b). Also, discretizing (3.24) using the quadrature rule (Baker 1978), which is applied in numerical integrations to achive high accuracy and to avoid the appearance of any singularities during the discretization processes, $\phi_{b}$ is determined from $(3.23 a)$. Note that $\phi_{S}^{\prime}(\tau(\delta ; 0))=0$ because no wave arrives on the shoreline at $t=0$. Lastly, convergence is checked through the relation between $u_{0}$ and $\phi_{b}$ shown in $(3.23 c)$.

To represent the shoreline motion in terms of $\phi_{S}$, equation (3.9) is utilized in the following analysis. Since $\phi_{S}$ and $A$ are related by

$$
\phi_{S}(\alpha)=A(2 \alpha)
$$

because $\alpha=\lambda / 2$ on the shoreline, equation (3.9) can be written by using the variables $\phi_{S}$ and $\alpha_{b}$ as follows:

$$
x_{S 0}=\frac{1}{8} \phi_{S}^{\prime}\left(\alpha_{b}(t)\right)-\frac{m}{128} \phi_{S}^{\prime \prime}\left(\alpha_{b}(t)\right)^{2}, \quad t_{S 0}=\frac{\alpha_{b}(t)}{m}-\frac{1}{8} \phi_{S}^{\prime \prime}\left(\alpha_{b}(t)\right),
$$

where the time variable $t$ introduced in the present section and $t_{S 0}$ in $\S 3.1$ are different because the former is based on the line $x=-r_{b}$ while the latter is the time on the shoreline as seen in figure 6. In the following, numerical results for the wave run-up and the back-wash are shown as an instructive example of the effectiveness of the present numerical method. The results are compared with the series solution in $\S 3.1$.

Since the value $\alpha$ is conserved on the incoming characteristics, it is calculated from 


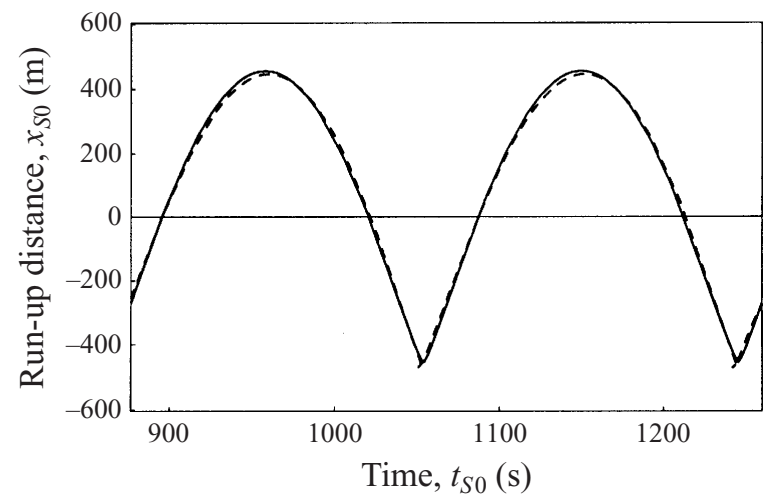

FiguRE 8. Run-up distance $x_{S 0}$ as a function of time $t_{S 0}$ due to the wave given by Carrier \& Greenspan's solution. Here, $T_{p}=191.6 \mathrm{~s}$ and $s_{l}=\frac{1}{20}$. Solid and broken lines indicate the exact and numerical solutions, respectively.

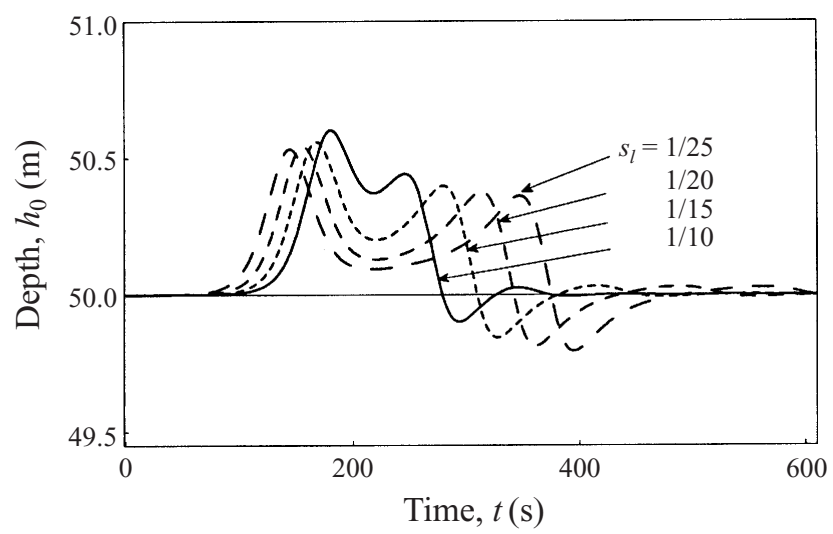

FIGURE 9. Water depth $h_{0}$ at $x=-r_{b}$ as a function of time $t$ due to solitary wave propagation. Here, $d=50 \mathrm{~m}, H / d=0.01$, and $s_{l}=\frac{1}{10}, \frac{1}{15}, \frac{1}{20}$, and $\frac{1}{25}$.

the incident wave, so that we have

$$
\begin{aligned}
\alpha_{b}(t) & =u_{0 I b}+2 \sqrt{g h_{0 I b}}+m t \\
& \approx \frac{2 c_{0}}{d} \zeta_{I}^{(1)}+2 \sqrt{g d}+m t,
\end{aligned}
$$

where the subscript $I$ of $u_{0 I b}$ and $h_{0 I b}$ indicates the values for the incident wave, and where the same parameters adopted in $\S 3.1$ are applied. In order to check the accuracy of the present numerical method, Carrier \& Greenspan's solution for the limiting wave

$$
\phi=\frac{8 g}{\omega} J_{0}\left(\frac{\omega \sigma}{2 m}\right) \sin \left(\frac{\omega \lambda}{2 m}\right)
$$

is compared to the numerical result, as shown in figure 8 , where $\omega=2 \pi / T_{p}, T_{p}=$ $191.6 \mathrm{~s}$, and $s_{l}=\frac{1}{20}$. The boundary condition of the numerical simulation is given at $x=-10000 \mathrm{~m}$, so that the second approximation in (3.28) has a high enough accuracy. On the other hand, the initial condition is given by $\beta_{b}=0$ and $\phi_{S}=0$. The result in figure 8 shows that the present method works reasonably well even for the critical case because the numerical result converges to the exact solution. 


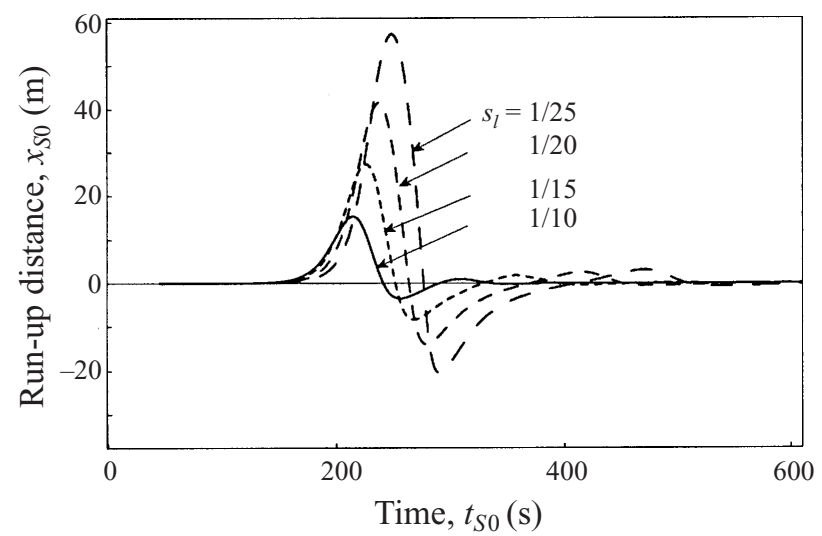

FIGURE 10. Run-up distance $x_{S 0}$ as a function of time $t_{S 0}$ due to solitary wave propagation. Here, $d=50 \mathrm{~m}, H / d=0.01$, and $s_{l}=\frac{1}{10}, \frac{1}{15}, \frac{1}{20}$, and $\frac{1}{25}$.

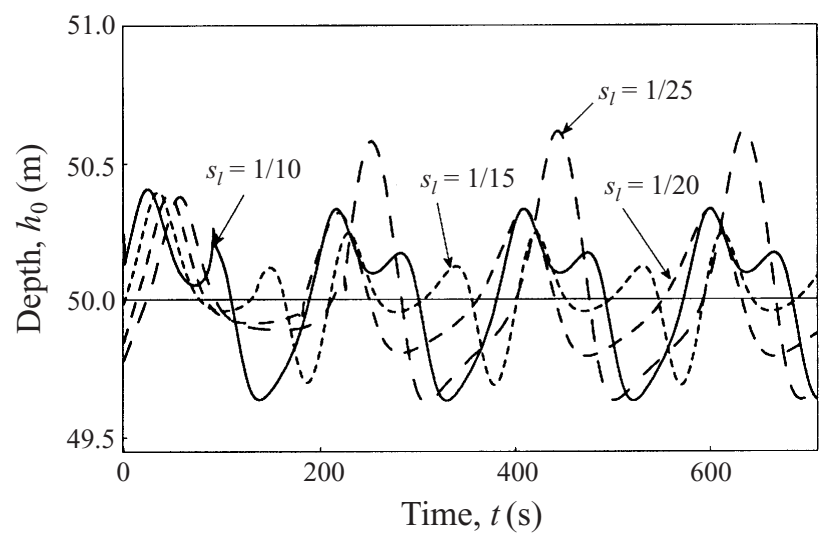

FiguRE 11. Water depth $h_{0}$ at $x=-r_{b}$ as a function of time $t$ due to cnoidal wave propagation. Here, $d=50 \mathrm{~m}, H / d=0.01$, and $s_{l}=\frac{1}{10}, \frac{1}{15}, \frac{1}{20}$, and $\frac{1}{25}$.

Figures 9 and 10 show the water depth at the boundary $x=-r_{b}$ and the run-up height of the solitary wave, respectively. The analytical solution in $\S 3.1$ can describe the wave profile in general, except the tails, as seen in figure 3 . The lowest trough in figure 10 shows the multiple-valued transformation between $(t, x)$ and $(\lambda, \sigma)$ of Carrier \& Greenspan's transformation as indicated by Stoker (1948). The sharp corner there corresponds to wave breaking during back-wash. Note that the integral equation (3.17) is applicable only to non-breaking waves.

Figures 11 and 12 show the water depth at the boundary $x=-r_{b}$ and the runup height of the cnoidal wave, respectively. The curve in figure 11 for $s_{l}=\frac{1}{10}$ is multi-valued around $t=100 \mathrm{~s}$, i.e. the curve wraps back on itself. Because the multi-valuedness of the curve indicates that the solution obtained from Carrier \& Greenspan's transformation does not describe physically-acceptable solutions, we need to check whether the multiplicity appears also in the beach region between the shoreline and the lower boundary of the beach. If it does not then we may apply Carrier \& Greenspan's solution for the wave motion.

Comparison between figure 12 and figure 4 shows that the wave profile for $s_{l}=\frac{1}{10}$ is quite similar for these figures. On the other hand, there is a discrepancy between those for $s_{l}=\frac{1}{15}, \frac{1}{20}, \frac{1}{25}$. The reason is that the convergence of the series (3.4) becomes worse 


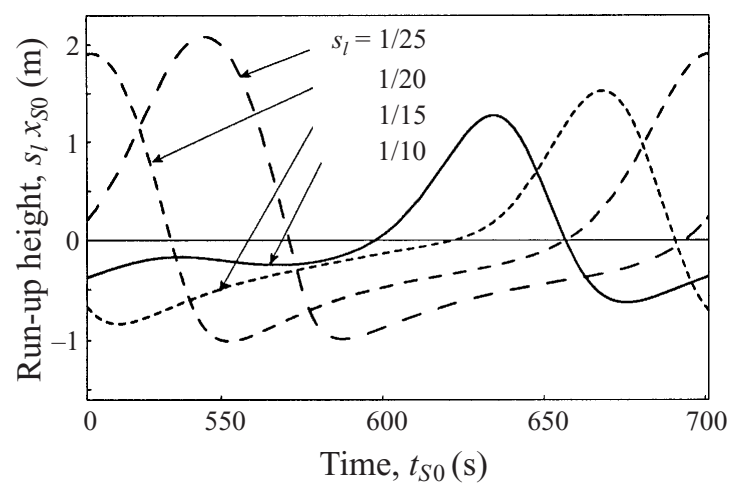

FIGURE 12. Run-up height as a function of time $t_{S 0}$ due to cnoidal wave propagation. Here, $d=50 \mathrm{~m}, H / d=0.01$, and $s_{l}=\frac{1}{10}, \frac{1}{15}, \frac{1}{20}$, and $\frac{1}{25}$.

as the distance from the shoreline to the edge of the slope, i.e. $r_{b}$, becomes longer. Nevertheless, the run-up height seems to be described in general by the analytical solution in $\S 3.1$ as shown in figure 5, where the data obtained from the exact integral formula and the series solution collapse onto each other as $\kappa$ becomes smaller, i.e. at $s_{l}=\frac{1}{10}$ and $\frac{1}{15}$.

\section{Obliquely incident waves over an uneven bed surface}

Waves incident obliquely on the beach induce a current because of the zigzag motion of the fluid particles in the swash zone (Asano 1994; Brocchini 1997). In this section, it is shown that bed unevenness strongly affects the current because of the thin sheet of liquid in the swash zone. We consider a bed which has a sinusoidal fluctuation with amplitude $0.5 \mathrm{~m}$ and wavelength $1000 \mathrm{~m}$ as an example. Here, $d=50 \mathrm{~m}$ and $H / d=0.01$, as in the previous section.

The series solution in $\S 3.1$ is applied in $\S 4.2$ for evaluation of the current because it can be treated analytically so that it is possible to proceed with the computations accurately without the need for a large computer memory. First, in $\S 4.1$, the solution of the one-dimensional NLSWE in $\S 3$ is extended to the solution of the twodimensional NLSWE by means of a geometrical considerations discussed below. Second, the solution $v_{1}$ which satisfies both the momentum equation (2.6) and the boundary condition at the edge of the sloping beach for the obliquely incident waves is derived. Then in $\S 4.2$, the mass flux and the time-averaged velocity of the cnoidal and the solitary waves are calculated from the formula derived in $\S 4.1$. In the following, the subscripts 0 and 1 of $b_{0}$ and $y_{1}$ are dropped for simplicity of the manipulations.

\subsection{Longshore velocity}

Since we are considering the quasi-normally incident waves, the governing equation of the zeroth order, that is (2.5), does not involve the $y$-derivative terms explicitly, but the variable $y$ enters the equation through the boundary condition at the edge of the sloping beach. Since the formula (2.14) is also satisfied for the quasi-normally incident waves with weak nonlinearity (Miles 1977), the extension of the matching condition for the one-dimensional waves to the two-dimensional waves is achieved by replacing the phase function of the incident wave in (3.8) as follows:

$$
\zeta_{0 b}+\frac{d}{c_{0}} u_{0 b}=\left.2 \psi\right|_{x=-r_{b}}, \quad \zeta_{I}^{(1)} \equiv \psi\left(-\omega t+k_{x} x+k_{y} y\right) .
$$


Here $\left(k_{x}, k_{y}\right)$ and $\omega$ are the wavenumber vector and the frequency, respectively, and $\psi$ is a function representing the two-dimensional incident wave. In the following, the parametric form of $u_{0}$ and $h_{0}$ with respect to the variable $y$ is determined through geometrical considerations.

In order to obtain the solution which satisfies the boundary condition (4.1), Carrier $\&$ Greenspan's transformation of the variables $x$ and $t$ is applied for the derivation. At first, based on the transformation given by (3.1), the bed level change due to the fluctuation from $z=s_{l} x$ to $z=s_{l} x+b(y)$ necessitates the replacement

$$
x \rightarrow X \equiv x+\frac{b(y)}{s_{l}}
$$

in (3.1), so that the shoreline is represented by $\sigma=0$. Next, in order that the wave crest at the end of the sloping beach is straight after the replacement (4.2), the variable $t$ also needs to be replaced as follows:

$$
t \rightarrow T \equiv t+\frac{1}{\omega}\left(k_{x} \frac{b(y)}{s_{l}}-k_{y} y\right) .
$$

As a result, we have an extended Carrier \& Greenspan's transformation which is applicable to waves of oblique incidence over a wavy bed. The weakly twodimensional solution given by $u_{0}=u_{0}(T, X)$ and $h_{0}=h_{0}(T, X)$ obviously satisfies (2.5) because (4.2) and (4.3) are a linear transformation of the variables $t$ and $x$. Also, it is shown that the above weakly two-dimensional solution is consistent with the boundary condition (4.1) since (4.1) is represented by $X$ and $T$ only.

Alongshore velocity $v_{1}$ is determined from (2.6c). Substitution of the expression $h_{0}=h_{0}(T, X)$ into this equation and the change of the variables $(x, t)$ to $(X, T)$ lead to

$$
\frac{\partial v_{1}}{\partial T}+u_{0} \frac{\partial v_{1}}{\partial X}=-\frac{b^{\prime}(y)}{s_{l}}\left(g \frac{\partial h_{0}}{\partial X}+g s_{l}\right)-\frac{g}{s_{l} \omega}\left(k_{x} b^{\prime}(y)-k_{y} s_{l}\right) \frac{\partial h_{0}}{\partial T},
$$

where the prime on $b(y)$ denotes its $y$-derivative. The first term of the right-hand side of (4.4) represents the external force due to the gravitational force produced by the bed slope, while the second term is related to the incident angle of the wave. The solution $v_{1}$ balancing these external forces is found as follows:

$$
v_{1}(t, x, y)=\frac{b^{\prime}(y)}{s_{l}} u_{0}(T, X)+\left(\sin \theta-\frac{b^{\prime}(y)}{s_{l}} \cos \theta\right) v_{p}(T, X),
$$

where $v_{p}$ is the particular solution of (4.4), which is in balance with the external force $\partial h_{0} / \partial T$ and has been found by Brocchini \& Peregrine (1996). Here the wavenumber vector is written in terms of the magnitude $k$ and the argument $\theta$ as

$$
\left(k_{x}, k_{y}\right)=k(\cos \theta, \sin \theta) \text {. }
$$

Also, the parametric representation of $u_{0}, h_{0}$, and $v_{p}$ in (4.5) is given by

$$
\begin{gathered}
u_{0}=\frac{m}{\sigma} \frac{\partial \phi}{\partial \sigma}, \quad h_{0}=\frac{s_{l}}{16 m} \sigma^{2}, \quad v_{p}=\frac{g k s_{l}}{4 \omega} \frac{\partial \phi}{\partial \lambda}, \\
x=-\frac{\sigma^{2}}{16 m}+\frac{1}{4} \frac{\partial \phi}{\partial \lambda}-\frac{u_{0}^{2}}{2 m}-\frac{b(y)}{s_{l}}, \\
t=\frac{\lambda}{2 m}-\frac{u_{0}}{m}-\frac{1}{C}\left(\frac{b(y)}{s_{l}} \cos \theta-y \sin \theta\right) .
\end{gathered}
$$

The introduction of the multiple scale in (2.4) is necessary to eliminate a secular 
term of the perturbation equations because the expansion of (4.1) with respect to $k_{y}$ produces a secularity which is proportional to the variable $y$.

Although the general solution of (4.4) can be determined from the initial condition of $v_{1}$, it has been ignored in (4.5). The shore current specified by the particular solution (4.5) is examined in the following section.

\subsection{Mass flux and time-averaged velocity over the beach}

In this section, the mass flux and the time-averaged flow velocity induced by a cnoidal wave, i.e. a periodic wave, is discussed first, and then the differences between the flow of cnoidal and solitary waves are studied. The series solution derived in $\S 3.1$ is used for evaluation of these vector fields. The effect of the bed topography on the shore current is calculated applying (4.5).

A mass flux of unit width of the cnoidal wave is defined by

$$
\begin{aligned}
q_{x} & =\frac{1}{T_{p}} \int_{0}^{T_{p}} u h \mathrm{~d} t, \quad q_{y}=\frac{1}{T_{p}} \int_{0}^{T_{p}} v h \mathrm{~d} t, \quad \text { for periodic waves } \\
u h & \approx u_{0} h_{0}+u_{2} h_{0}+u_{0} h_{2}, \quad v h \approx v_{1} h_{0}, \\
\phi & \approx \phi_{a p},
\end{aligned}
$$

where $T_{p}$ denotes the wave period. Since $q_{y}$ is known by (4.5), $q_{x}$ can be calculated by the mass conservation law (2.7), which yields

$$
q_{x}=-\int_{x_{\text {Smax }}}^{x} \frac{\partial q_{y}}{\partial y} \mathrm{~d} x
$$

Here, $x_{\operatorname{Smax}}$ denotes the maximum run-up distance from the shoreline, where it is clear that $q_{x}=0$. Substitution of $v_{1}$ of (4.5) into the expression for $q_{y}$ gives

$$
q_{y}=\left(\sin \theta-\frac{b^{\prime}(y)}{s_{l}} \cos \theta\right) q_{y 0}, \quad q_{y 0} \equiv \frac{1}{T_{p}} \int_{0}^{T_{p}} \frac{g k s_{l}}{4 \omega} \frac{\partial \phi}{\partial \lambda} h_{0} \mathrm{~d} t,
$$

because of the zeroth-order mass conservation law $\overline{u_{0} h_{0}}=0$, where the over bar denotes the time average. Equations (4.9) and (4.10) indicate that the mass flux due to the second-order effect of the flow with respect to the bed fluctuation can be evaluated without first obtaining $h_{2}$ and $u_{2}$.

In this section, the time integration at a fixed spatial position is achieved numerically as follows. First, $\sigma$ is determined as a function of $\lambda$ for fixed values of $X$ through the transformation of $x$ in (4.7), which leads to $\sigma=\sigma(\lambda ; X)$. Second, this relation is substituted into the transformation of $t$ in (4.7). Then, all the variables in (4.7) are represented as a function of $t$ through the intermediate variable $\lambda$.

The parameters are fixed to $d=50 \mathrm{~m}, H / d=0.01$, and $T_{p}=191.6 \mathrm{~s}$, i.e. $\mu=0.99$. To investigate the basic property of the wave-induced motion, we will start with a discussion of the flow over a uniformly sloping beach, i.e. $b(y)=0$. Figures 13(a) and $13(b)$ show the mass flux $q_{y 0}$ and the mean velocity $\bar{v}_{p}$ due to the cnoidal wave, respectively. The crests of these curves show that the mass flux attains its maximum at the lowest part of the swash zone, while the mean velocity takes its maximum at the shoreline of still water.

Next, in order to see the difference between $\bar{u}_{0}$ and $\bar{v}_{p}$, both curves are plotted simultaneously in figure $14(a) ; \bar{u}_{0}$ is negligible compared to $\bar{v}_{p}$ as implied by the fact that the flow velocity normal to the shoreline is almost time periodic, while the fluid particles are drifting alongshore in a zigzag motion. The overall picture of the mass 

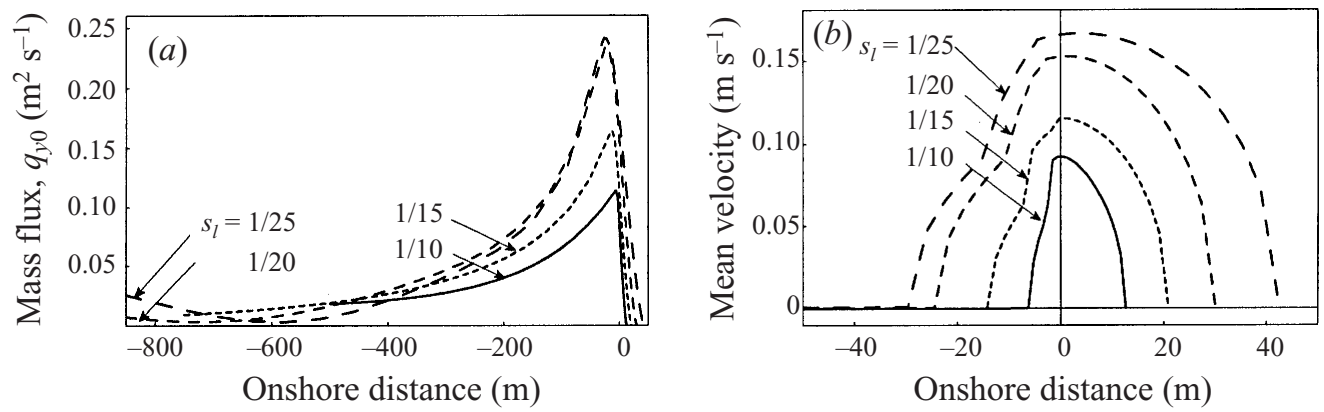

FIGURE 13. Mass flux and time-averaged velocity due to the cnoidal wave for $b(y)=0$.

(a) $q_{y 0}$ and $(b) \bar{v}_{p}$, for $s_{l}=\frac{1}{10}, \frac{1}{15}, \frac{1}{20}$, and $\frac{1}{25}$.
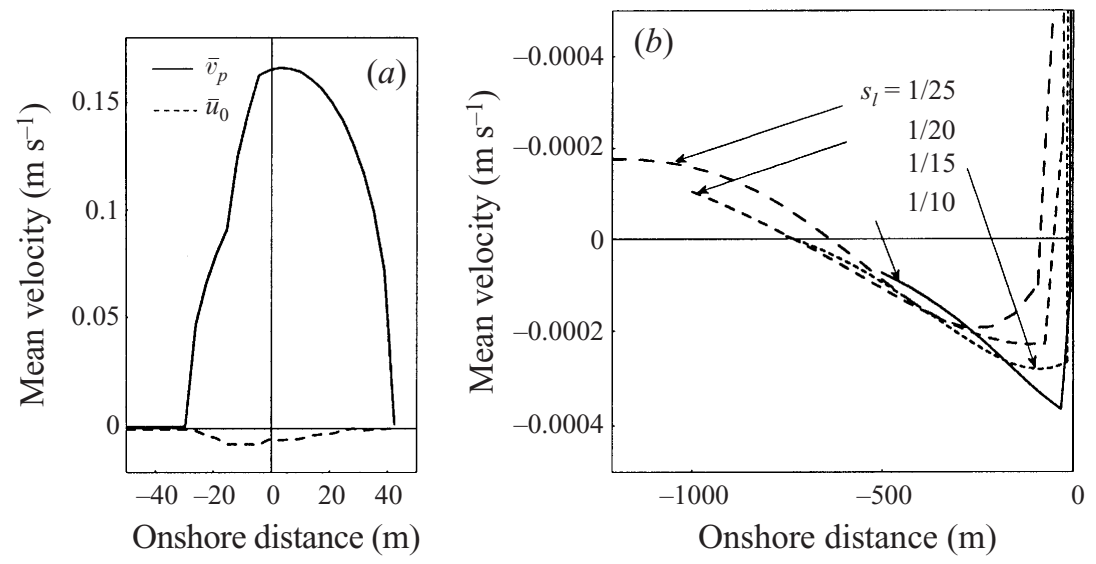

FIGURE 14. Detailed picture of time-averaged velocity. $(a) \bar{v}_{p}$ and $\bar{u}_{0}$ for $s_{l}=\frac{1}{25}$.

(b) Mean velocity $\bar{v}_{p}$ outside the swash zone.

flux and the mean velocity in figures 13 and 14 is quite similar to the work done by Brocchini \& Peregrine (1996). Kobayashi \& Karjadi (1994) measured longshore currents and calculated nonlinear shallow water equations having bottom friction terms. Their experiment shows that the peak of the longshore currents occurs near the shoreline of the still water, while their numerical simulation shows that the peak position is shifted off-shoreward. This discrepancy implies that the bottom friction in the swash zone is overestimated in their study.

Because a time-average of nearly sinusoidal oscillations is estimated by the mean value between the maximum and the minimum of the displacement, the order of $\bar{v}_{p}$ in the present study is also estimated as follows:

$$
\begin{aligned}
O\left(\bar{v}_{p}\right) & =\frac{1}{2}\left(\max _{\lambda}\left[\left.v_{p}\right|_{\sigma=0}\right]+\min _{\lambda}\left[\left.v_{p}\right|_{\sigma=0}\right]\right) \\
& =\frac{g k}{2 \omega}\left(s_{l} x_{S 0 \max }+s_{l} x_{S 0 \min }\right) \\
& =\frac{g H}{8 C}\left\{f_{\max }(\kappa, \mu)+f_{\min }(\kappa, \mu)\right\},
\end{aligned}
$$

where (3.10) and the expression for $v_{p}$ in (4.7) have been used in the manipulation. Plugging in the values for $f_{\max }$ and $f_{\min }$ from figure 5 to (4.11), it can be verified that the order of $\bar{v}_{p}$ is well-estimated by (4.11). 


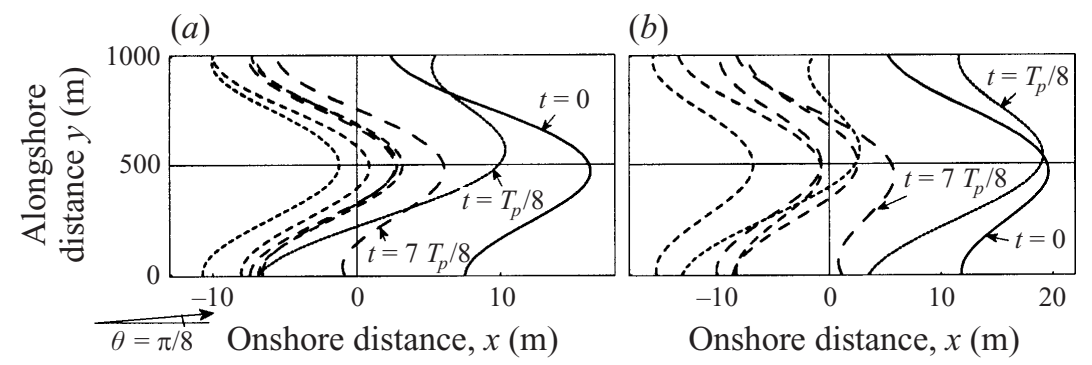

FIGURE 15. Shoreline profile $x_{S 0}$ as a function of $y$ at $t=0, T_{p} / 8, \ldots$, and $7 T_{p} / 8$. Here, $\theta=\pi / 8$. (a) $s_{l}=\frac{1}{10},(b) s_{l}=\frac{1}{25}$. The length of the dashes for each curve indicates the time elapsed from $t=0$. For example, the full solid line depicts the run-up distance at $t=0$ the dots to $T_{p} 18$, and the longest dashes correspond to the curve at $t=7 T_{p} / 8$.

In figure $14(b)$, the distribution of $\bar{v}_{p}$ outside the swash zone is depicted for a discussion of the qualitative difference between the mass flux and the time-averaged velocity. The mass flux $q_{y 0}$ is positive in the swash zone as seen in figure 13(b), whereas the time-averaged velocity $\bar{v}_{p}$ becomes negative outside the swash zone. In addition, the maximum of $q_{y 0}$ and the minimum of $\bar{v}_{p}$ seem to be reached at the lower boundary of the swash zone. Though these values are quite small for the small-amplitude wave with $H / d=0.01$, their magnitude will become prominent for large-amplitude waves.

Now, consider the fluctuating bed level given by

$$
b(y)=b_{a m} \cos \left(\frac{2 \pi y}{L_{b}}\right) .
$$

Here $b_{a m}$ and $L_{b}$ denote the bed amplitude and the wavelength along the $y$-direction. In the following, $L_{b}=1000 \mathrm{~m}$ and $b_{a m}=0.5 \mathrm{~m}$. A parametric representation of the moving shoreline at the zeroth order of $\epsilon$ is given by $\left(\left.t\right|_{\sigma=0},\left.x\right|_{\sigma=0}\right)$ in (4.7). Figure 15 depicts the shoreline generated by obliquely incident waves with $\theta=\pi / 8$ as a function of $y$, at $t=0, T_{p} / 8, \ldots$, and $7 T_{p} / 8$, successively. The peak of the shoreline profile during the run-up is located on the lower side of the bed trough, i.e. $y \leqslant L_{b} / 2=500 \mathrm{~m}$, and it moves to the upper side, i.e. above $y=500 \mathrm{~m}$, during the back-wash because of the gravity force due to the bed slope. On the other hand, the run-up height, which is seen from figure 15 as the envelope of the upper shorelines, is the same as, that for the wave over the uniformly sloping beach except for geometrical translation corresponding to the translation of the bed level. The effect of $v_{1}$ on the shoreline profile will appear in the second order term of the $\epsilon$-perturbation as discussed in $\S 2.1$.

The mass flux $\left(q_{x}, q_{y}\right)$ and the time-averaged velocity $\left(\bar{u}_{0}, \bar{v}_{1}\right)$ generated by the waves over the fluctuating bed are shown in figures 16, 17, and 18. Here the bed slope $s_{l}$ is fixed to $\frac{1}{25}$. The ridge and the trough of the beach correspond to $y=0 \mathrm{~m}$ and $y=500 \mathrm{~m}$, respectively. The arrows and the dotted line at $x=-29.6 \mathrm{~m}$ in these figures show the direction of the vector and the mean shoreline at the lowest side of the swash zone, respectively. The magnitude of the vectors can be estimated from (4.5), (4.10), and figure 13. These figures imply that the bed unevenness, where the fluctuation of the bed slope alongshore has the order $b_{a m} / L_{b}=1 / 2000$, causes a meandering of these vector fields. Figure 16 shows the vector fields generated by the normally incident waves. The strong mass flux outside the swash zone is induced by the existence of the swash zone as shown in figure $16(a)$. The inhomogeneity of the alongshore mass flux generates offshore currents because of the continuity condition (4.9) for the fluid mass. Because the water depth near the shoreline is small compared 
(a)

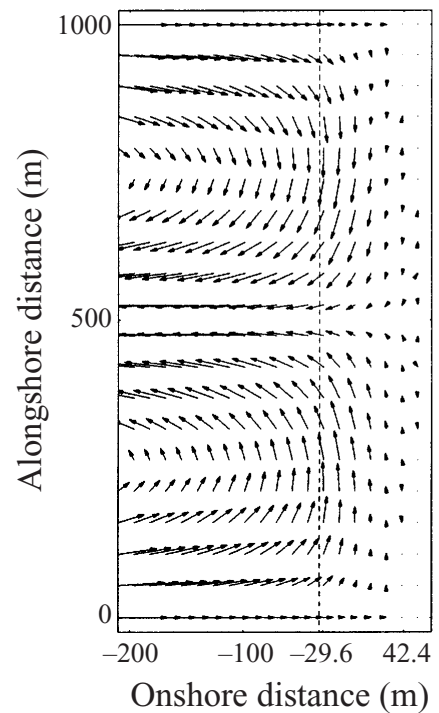

(b)

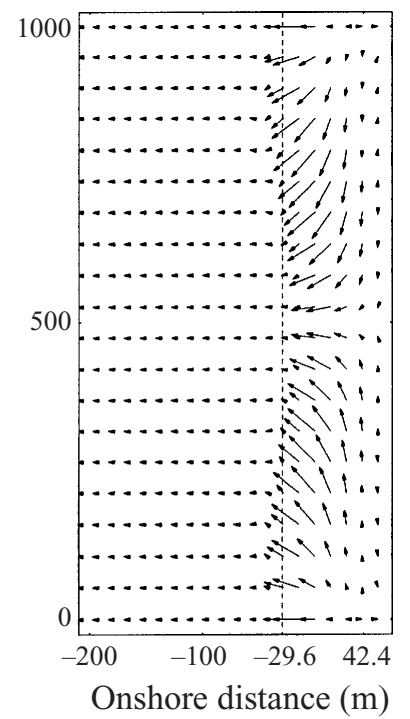

FIGURE 16. (a) Mass flux $\left(q_{x}, q_{y}\right)$ and $(b)$ time-averaged velocity $\left(\bar{u}_{0}, \bar{v}_{1}\right)$ due to the cnoidal wave for $s_{l}=\frac{1}{25}, b_{a m}=0.5 \mathrm{~m}$, and $\theta=0$.

(a)

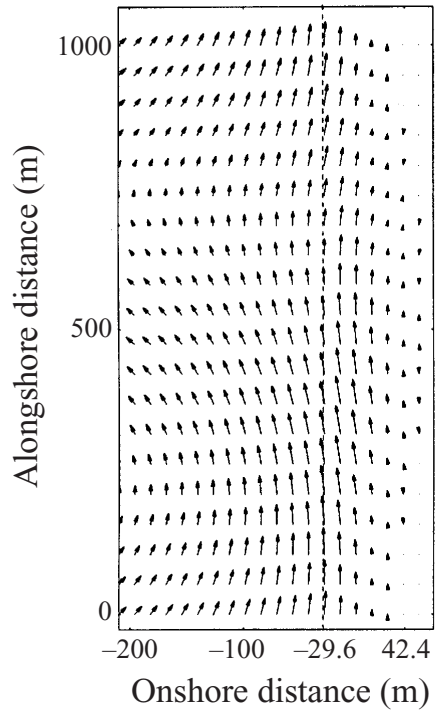

(b)

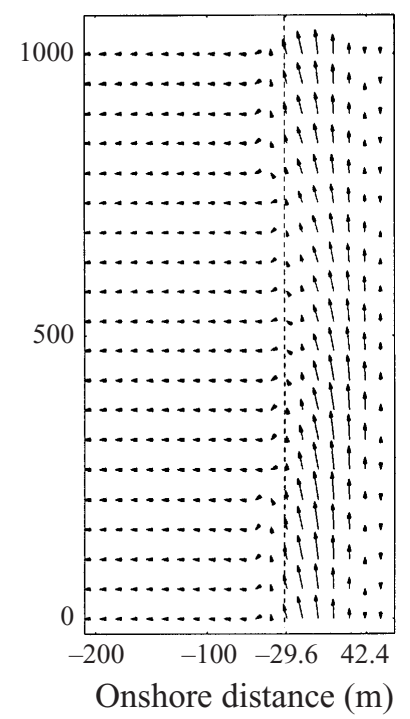

Figure 17. (a) Mass flux $\left(q_{x}, q_{y}\right)$ and $(b)$ time-averaged velocity $\left(\bar{u}_{0}, \bar{v}_{1}\right)$ due to the cnoidal wave for $s_{l}=\frac{1}{25}, b_{a m}=0.5 \mathrm{~m}$, and $\theta=\pi / 8$.

to the bed level fluctuation, $\bar{v}_{1}$ is strongly influenced by the bed unevenness as seen in figure $16(b)$.

In figure 17 , the incident wave angle is fixed to $\theta=\pi / 8$ to see the effect of the incident wave angle on the flow. While the obliquely incident wave generates a large time-averaged velocity in the alongshore direction in the swash zone, the velocity outside is not large. 
(a)

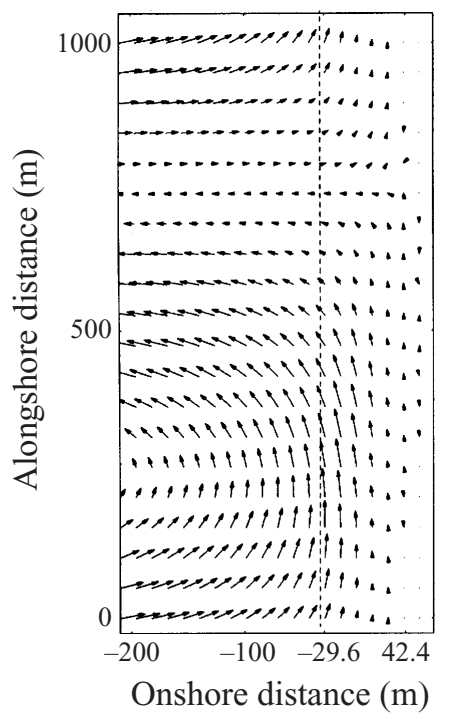

(b)

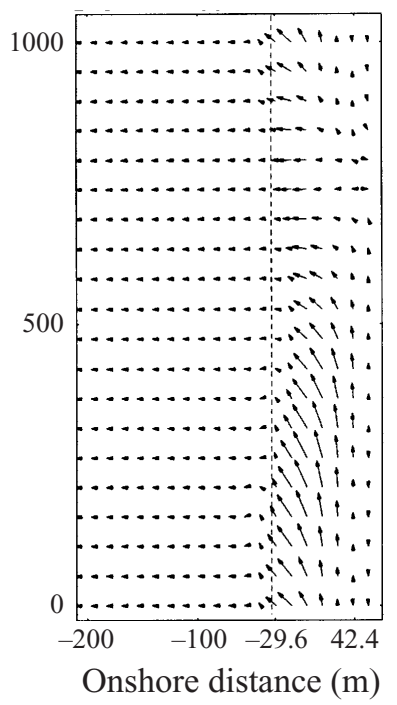

Figure 18. (a) Mass flux $\left(q_{x}, q_{y}\right)$ and $(b)$ time-averaged velocity $\left(\bar{u}_{0}, \bar{v}_{1}\right)$ due to the cnoidal wave under the critical condition. Here, $s_{l}=\frac{1}{25}, b_{a m}=0.5 \mathrm{~m}$, and $\theta_{c r}=0.025 \pi$.

(a)

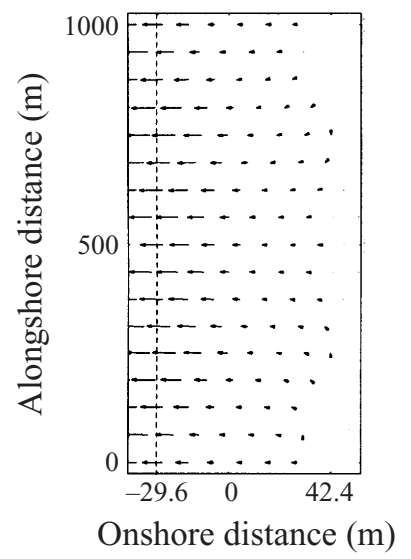

(b)

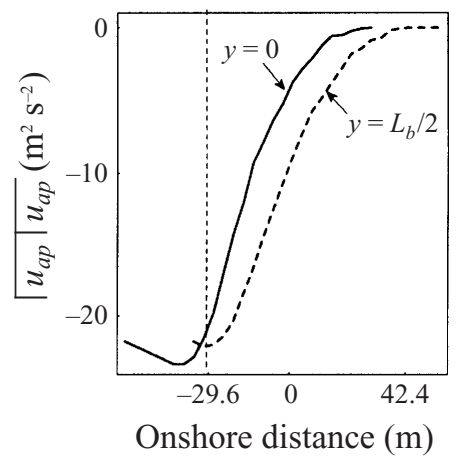

FIGURE 19. Distribution of the time average of $\left|\boldsymbol{u}_{a p}\right| \boldsymbol{u}_{a p}:(a)$ vector fields and $(b)$ value. Here, $b_{a m}=0.5 \mathrm{~m}, \theta=\pi / 8$, and $s_{l}=\frac{1}{25}$.

Figure 18 shows the critical mass flux due to the obliquely incident waves, where the net longshore mass flux disappears because of the component of gravity in the alongshore direction. This critical condition is derived from (4.10), which leads to

$$
\left.q_{y}\right|_{\theta=\theta_{c r}}=0, \quad \text { where } \tan \theta_{c r}=\frac{\max _{y} b^{\prime}(y)}{s_{l}} .
$$

Note that the alongshore velocity $\bar{v}_{1}$ is not equal to 0 at the boundary where $q_{y}=0$, as implied by the expression (4.5) for $\bar{v}_{1}$.

Though we ignored the bottom friction for evaluation of the flow velocity, a 
(a)

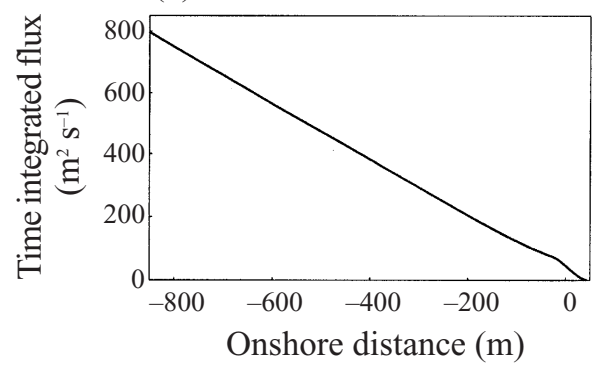

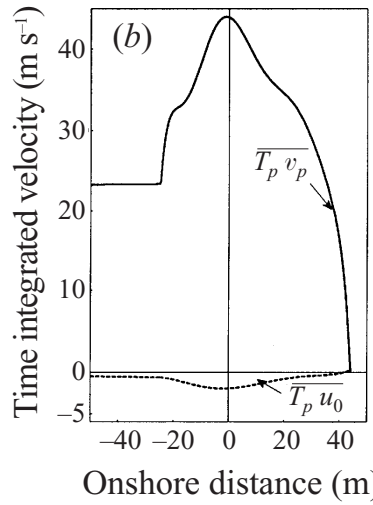

FIGURE 20. Time-integrated mass flux and velocity due to the solitary wave for $b(y)=0, s_{l}=\frac{1}{25}$, and $H / d=0.01$. (a) $T_{p} q_{y 0}$. (b) $\overline{T_{p} u_{0}}$ and $\overline{T_{p} v_{p}}$ for $s_{l}=\frac{1}{25}$. The coordinates $x=-24.9 \mathrm{~m}$ and $44.2 \mathrm{~m}$ show the minimum and the maximum run-up distances, respectively.

qualitative discussion of the bottom friction is possible through

$$
\overline{\left|\boldsymbol{u}_{a p}\right| \boldsymbol{u}_{a p}}, \quad \boldsymbol{u}_{a p} \equiv\left(u_{0}, v_{1}\right) .
$$

Figure 19 shows the distribution of the vector $\overline{\left|\boldsymbol{u}_{a p}\right| \boldsymbol{u}_{a p}}$ in the swash zone due to the cnoidal wave for $s_{l}=\frac{1}{25}, \theta=0$, and $b_{a m}=0.5 \mathrm{~m}$. All of the vectors are pointing offshore, similar to the time-averaged velocity vector $\overline{\boldsymbol{u}}_{a p}$ shown in figure $16(b)$. The sign of the $y$-component in the two figures seems to be identical, which can be explained if the approximation

$$
\boldsymbol{u}_{a p} \approx\left(\tilde{u}_{0}, \bar{v}_{1}\right), \quad \tilde{u}_{0} \equiv u_{0}-\bar{u}_{0}
$$

is applied to (4.14), as pointed out by Mei (1983), as follows:

$$
\overline{\left|\boldsymbol{u}_{a p}\right| \boldsymbol{u}_{a p}} \approx \overline{\sqrt{\tilde{u}_{0}^{2}+\bar{v}_{1}^{2}}\left(\tilde{u}_{0}, \bar{v}_{1}\right)} \approx \overline{\left|\bar{v}_{1}\right|\left(\tilde{u}_{0}, \bar{v}_{1}\right)}=\left|\bar{v}_{1}\right|\left(0, \bar{v}_{1}\right) .
$$

Figure 19(b) implies that the peak of the bottom friction appears near the foreshore at $x=-29.6 \mathrm{~m}$ in the swash zone.

Finally, figure 20 depicts the time-integrated mass flux and velocity of the solitary wave, which are defined by

$$
\begin{array}{ll}
T_{p} q_{x}=\int_{0}^{T_{p}} u h \mathrm{~d} t, & T_{p} q_{y}=\int_{0}^{T_{p}} v h \mathrm{~d} t, \\
\overline{T_{p} u_{0}} & =\int_{0}^{T_{p}} u_{0} \mathrm{~d} t, \quad \overline{T_{p} v_{1}}=\int_{0}^{T_{p}} v_{1} \mathrm{~d} t,
\end{array}
$$

respectively, where $T_{p}$ is multiplied by the mass flux and the time-averaged velocity because the time average of these values approaches 0 as $T_{p} \rightarrow \infty$. Here the slope and the wave amplitude are fixed to $s_{l}=\frac{1}{25}$ and $H / d=0.01$, respectively. For a period of $T_{p}=191.6 \mathrm{~s}$ for the cnoidal wave, the time-integrated mass flux and velocity of the solitary wave are roughly two times larger than that of the cnoidal wave. The fluctuations in these curves come from the tails of the solitary wave. As noted in §3.2, these fluctuations have not been accurately described by the truncated series solution obtained in $\S 3.1$ since the series could not reproduce the tails of the solitary wave. Figure 21 shows the time-integrated mass flux and velocity fields of the solitary wave for oblique incidence with the angle $\theta=\pi / 8$. The solitary wave transports mass in a 
(a)

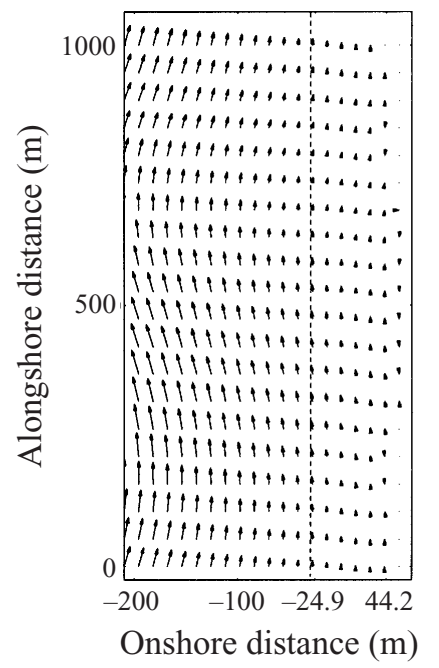

$(b)$

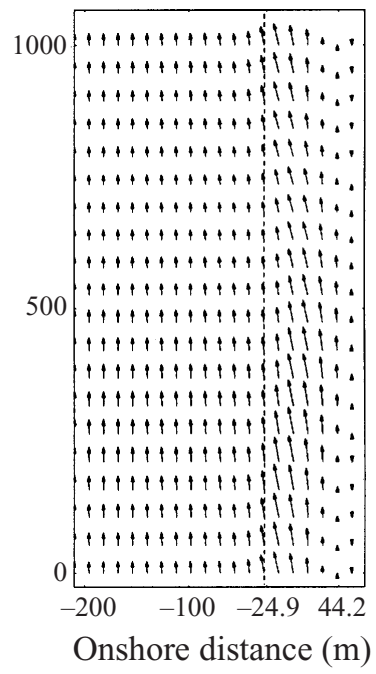

FIgURE 21. (a) Time-integrated mass flux and $(b)$ velocity fields for the solitary wave. Here, $b_{a m}=0.5 \mathrm{~m}, \theta=\pi / 8, s_{l}=\frac{1}{25}$, and $H / d=0.01$.

uniform direction over the whole region of the beach, and all the velocity vectors are directed alongshore, because the flow is not oscillatory.

\section{Concluding remarks}

Run-up and back-wash of the cnoidal and the solitary waves are calculated for a finite, 'wavy-bottom' beach with an ocean basin of constant depth. It is applied to large swash motions, where the swash amplitude is comparable to the beach length. An exact integro-differential equation derived by Riemann's method is introduced and solved numerically. Since it is satisfied at the boundary between the beach and the ocean basin, the calculation is performed along this boundary, and Riemann's representation provides an efficient method for calculating non-breaking long-wave motions for various kinds of incident waves. The comparison between the series solution and the solution of the integral equation reveals that the swash motion due to non-breaking waves is well predicted by the series solution, provided that the nonlinearity of the waves at the matching point, i.e. at the lower boundary of the beach, is negligible.

A 'weakly two-dimensional solution' describes the flow induced by obliquely incident waves over the spatially fluctuating bed topography. The shoreline motion, the mass flux and the time-averaged velocity influenced by the bed unevenness are evaluated systematically. The time-averaged flow velocity in the swash zone is dominated by the bed level change since the water depth there is comparable to the bed amplitude. A strong offshore mass flux outside the swash zone is generated by the inhomogeneity of the alongshore current caused by the gravity force due to the wavy bed. The solitary wave runs up the beach and induces an alongshore current more strongly than the cnoidal wave because the solitary wave has a non-zero mass transport velocity.

Bottom friction is qualitatively discussed using the flow velocity calculated from 
the inviscid two-dimensional NLSWE. The peak of the bottom friction appears near the foreshore in the swash zone.

Though we have only studied currents over a sinusoidal bed surface, various bottom topographies could be investigated if $b(y)$ is expanded in a Fourier series.

This work was partly completed during the first author's stay at the Asian Institute of Technology, Bangkok, Thailand, from 1995 to 1997. Discussions with Dr Sutat Weesakul are much appreciated. The authors are also grateful to Dr Anil C. Wijeyewickrema, Mr Pranab Jyoti Baruah, and Mr Tom Copeland for checking the manuscript.

\section{REFERENCES}

Asano, T. 1994 Swash motion due to obliquely incident waves. Proc. 24th Intl Conf. on Coastal Engineering, Kobe, pp. 27-41. ASCE.

BAKER, T. H. 1978 The Numerical Treatment of Integral Equations. Oxford.

Brocchini, M. 1997 Eulerian and Lagrangian aspects of the longshore drift in the surf and swash zones. J. Geophys. Res. 102, 23155-23168.

Brocchini, M. \& Peregrine, D. H. 1996 Integral flow properties of the swash zone and averaging. J. Fluid Mech. 317, 241-273.

Carrier, G. F. \& Greenspan, H. P. 1958 Water waves of finite amplitude on a sloping beach. $J$. Fluid Mech. 4, 97-109.

Courant, R. \& Hilbert, D. 1965 Methods of Mathematical Physics II. Interscience Publishers.

Fuji, S. \& Күотон, H. 1998 Interaction of longshore currents in surf- and swash-zones. Proc. Third Intl Conf. on Hydrodynamics, Korea, pp. 285-290.

Ho, D. V. \& Meyer, R. E. 1962 Climb of a bore on a beach. Part 1. Uniform slope beach. J. Fluid Mech. 14, 305-318.

Kim, S. K., LiU, P. L. -F. \& LigGetT, J. A. 1983 Boundary integral equation solutions for solitary wave generation, propagation and run-up. Coastal Engng 7, 299-317.

Kobayashi, N. \& KarJadi, E. A. 1994 Swash dynamics under obliquely incident waves. Proc. 24th Intl Conf. on Coastal Engineering, Kobe, pp. 2155-2169. ASCE.

Lapidus, L. \& Pinder, G. F. 1982 Numerical Solution of Partial Differential Equations in Science and Engineering. John Wiley \& Sons.

MeI, C. C. 1983 The Applied Dynamics of Ocean Surface Waves. John Wiley and Sons.

Miles, J. M. 1977 Obliquely interacting solitary waves. J. Fluid Mech. 79, 157-169.

RozDESTVENSKII \& JANENKO, N. N. 1983 Systems of Quasilinear Equations and their Applications to Gas Dynamics. Translation of Mathematical Monographs, vol. 55. American Mathematical Society.

RYRIE, S. C. 1983 Longshore motion generated on beaches by obliquely incident bores. J. Fluid Mech. 129, 193-212.

Shen, M. C. \& Meyer, R. E. 1962 Climb of a bore on a beach, Part 3. Run-up. J. Fluid Mech. 16, $113-125$.

Stoker, J. J. 1948 The formation of breakers and bores. Commun. Pure Appl. Maths 1, 1-87.

Synolakis, C. E. 1987 The runup of solitary waves. J. Fluid Mech. 185, 523-545.

Synolakis, C. E., Deb, M. K. \& SkJelbreia, J. E. 1988 The anomalous behavior of the runup of cnoidal waves. Phys. Fluids 31, 3-5.

Wiegel, R. L. 1960 A presentation of cnoidal wave theory for practical application. J. Fluid Mech. 7, 273-286.

ZELT, J. A. 1991 The run-up of nonbreaking and breaking solitary waves. Coastal Engng 15, 205-246. 